\title{
Analysis of the Refined CRUST1.0 Crustal Model and its Gravity Field
}

\author{
Robert Tenzer • Wenjin Chen • Dimitrios Tsoulis • Mohammad Bagherbandi • \\ Lars E. Sjöberg • Pavel Novák • Shuanggen Jin
}

Received: 13 January 2014/ Accepted: 21 July 2014/Published online: 20 August 2014

(C) Springer Science+Business Media Dordrecht 2014

\begin{abstract}
The global crustal model CRUST1.0 (refined using additional global datasets of the solid topography, polar ice sheets and geoid) is used in this study to estimate the average densities of major crustal structures. We further use this refined model to compile the gravity field quantities generated by the Earth's crustal structures and to investigate their spatial and spectral characteristics and their correlation with the crustal geometry in context of the gravimetric Moho determination. The analysis shows that the average crustal density is $2,830 \mathrm{~kg} / \mathrm{m}^{3}$, while it decreases to $2,490 \mathrm{~kg} / \mathrm{m}^{3}$ when including the seawater. The average density of the oceanic crust (without the seawater) is $2,860 \mathrm{~kg} / \mathrm{m}^{3}$, and the average continental crustal density (including the continental shelves) is $2,790 \mathrm{~kg} / \mathrm{m}^{3}$. The correlation analysis reveals that the gravity field corrected for major known anomalous crustal density structures has a maximum (absolute) correlation with the Moho geometry. The Moho signature in these gravity data is seen mainly at the long-to-medium
\end{abstract}

R. Tenzer $(\bowtie) \cdot$ W. Chen

The Key Laboratory of Geospace Environment and Geodesy, School of Geodesy and Geomatics, Wuhan University, 129 Luoyu Road, Wuhan 430079, China

e-mail: rtenzer@sgg.whu.edu.cn

D. Tsoulis

Department of Geodesy and Surveying, Aristotle University of Thessaloniki, 54124 Thessaloniki, Greece

M. Bagherbandi · L. E. Sjöberg

Division of Geodesy and Geoinformatics, Royal Institute of Technology (KTH), Stockholm, Sweden

M. Bagherbandi

Department of Industrial Development, IT and Land Management, University of Gävle, Gävle, Sweden

P. Novák

New Technologies for the Information Society (NTIS), Faculty of Applied Sciences, University of West Bohemia, Plzeň, Czech Republic

S. Jin

Shanghai Astronomical Observatory, Chinese Academy of Sciences, 80 Nandan Road, Shanghai, China 
wavelengths. At higher frequencies, the Moho signature is weakening due to a noise in gravity data, which is mainly attributed to crustal model uncertainties. The Moho determination thus requires a combination of gravity and seismic data. In global studies, gravimetric methods can help improving seismic results, because (1) large parts of the world are not yet sufficiently covered by seismic surveys and (2) global gravity models have a relatively high accuracy and resolution. In regional and local studies, the gravimetric Moho determination requires either a detailed crustal density model or seismic data (for a combined gravity and seismic data inversion). We also demonstrate that the Earth's long-wavelength gravity spectrum comprises not only the gravitational signal of deep mantle heterogeneities (including the core-mantle boundary zone), but also shallow crustal structures. Consequently, the application of spectral filtering in the gravimetric Moho determination will remove not only the gravitational signal of (unknown) mantle heterogeneities, but also the Moho signature at the long-wavelength gravity spectrum.

Keywords Correlation $\cdot$ Crust $\cdot$ Density $\cdot$ Gravity $\cdot$ Mantle $\cdot$ Moho

\section{Introduction}

Methods for a spherical harmonic analysis and synthesis of the gravity field are often applied in global studies investigating the Earth's lithosphere structure (cf. Tsoulis 2004a, b; Tenzer et al. 2009a, 2012a, b, c, and references therein). These methods utilize a spectral representation of the Earth's external gravity field in terms of spherical harmonics available to a certain degree of spectral resolution. The same principle can be used in describing the gravity field quantities generated by the Earth's inner density structure. For this purpose, the gravimetric forward modelling is directly applied to compute the gravitational contributions of known density structures by using discrete data, which are primarily obtained from results of seismic surveys. Alternatively, the spherical harmonic analysis of these data is applied in this study to generate spherical coefficients, which describe a density structure in a spectral domain. These spherical coefficients are then utilized to evaluate the gravitational contributions of respective density structures inside the Earth.

Several different global models describing the Earth's structure by means of seismic velocities and/or mass density were developed based on the analysis of available seismic data. Dziewonski et al. (1975) introduced the parametric Earth models (PEM) consisting of piece-wise continuous analytical functions of the radial density and velocity variations defined individually for the oceanic (PEM-O) and continental (PEM-C) lithosphere down to a depth of $420 \mathrm{~km}$; below this depth, these models are identical. They also provided an averaged function for the whole lithosphere (PEM-A). Dziewonski and Anderson (1981) presented the preliminary reference Earth model (PREM), which provides information on the seismic velocities and density structure within the whole Earth's interior (including the core and mantle) by means of spherically homogenous stratigraphic layers. This model also incorporates the non-elastic dispersion and anisotropy, and it is thus frequency-dependent and transversely isotropic within the upper mantle. Kennett and Engdahl (1991) compiled the parameterized velocity model IASP91 that summarized travel time characteristics of main seismic phases. Kennett et al. (1995) compiled the AK135-f model by augmenting the AK135 velocity model with the density and Q model of Montagner and Kennett (1995). van der Lee and Nolet (1997) prepared the 1-D averaged model MC35 based on the PEM$\mathrm{C}$, while replacing the high- and low-velocity zones of the PEM-C by a constant S-wave 
velocity of $4.5 \mathrm{~km} / \mathrm{s}$ within the upper mantle down to a depth of $210 \mathrm{~km}$. Kustowski et al. (2008b) derived the transversely isotropic reference Earth model STW105. More recently, Simmons et al. (2010) developed the GyPSuM tomographic model of the mantle (P and S) seismic velocities and density through a simultaneous inversion of seismic body wave travel times and geodynamic observables including the free air gravity anomalies, tectonic plate divergence, dynamic surface topography and the excess ellipticity of the core-mantle boundary. They also incorporated mineral physics constraints in order to link seismic velocities and wave speeds with an underlying hypothesis that temperature is a principal cause of heterogeneities in the non-cratonic mantle. In addition to these Earth's synthetic models, several other global and regional seismic velocity models were developed. A summary of these models can be found in Trabant et al. (2012). For more details, we refer readers also to studies by Grand et al. (1997), Megnin and Romanowicz (2000), Grand (2002), Gung and Romanowicz (2004), van der Lee and Frederiksen (2005), Panning and Romanowicz (2006), Houser et al. (2008), Kustowski et al. (2008a, 2008b), Bedle and van der Lee (2009), Panning et al. (2010), Obrebski et al. (2010, 2011), Porritt et al. (2011), James et al. (2011), Lekic and Romanowicz (2011), Simmons et al. (2012), and others.

The PEM and PREM models provide 1-D density information only. Models based on a stratigraphic layering with a variable depth, thickness and lateral density distribution obviously represent the Earth's interior more realistically. Currently, available global models provide information on a 3-D density structure only within the crust and upper mantle. Note that despite Simmons et al. (2010) derived a 3-D density structure within the whole mantle, these data were not yet released publically. Nataf and Ricard (1996) derived the global model of the crust and upper mantle density structure based on the analysis of seismic data and additional constrains such as heat flow and chemical composition. Mooney et al. (1998) compiled the global crustal model with a $5 \times 5$ arc-deg spatial resolution. More recently, the global crustal model CRUST2.0 was compiled with a $2 \times 2$ arc-deg spatial resolution (Bassin et al. 2000). This model was prepared and administered by the U.S. Geological Survey and the Institute for Geophysics and Planetary Physics at the University of California. Both models were compiled based on seismic data published until 1995 and a detailed compilation of the ice and sediment thickness. The most recent version, the CRUST1.0, has a $1 \times 1$ arc-deg spatial resolution (Laske et al. 2012). The CRUST1.0 consists of the ice, water (upper, middle and lower), sediments (upper, middle and lower) and consolidated (crystalline) crustal layers. In addition, the lateral density structure of the upper mantle was incorporated into the CRUST2.0 and CRUST1.0 models. The globally averaged data from active seismic methods and deep drilling profiles were used to predict the sediment and crustal structure, where no seismic measurements were available (most of Africa, South America, Greenland and large parts of the oceanic lithosphere) by a generalization to similar geological and tectonic settings. Despite the density distribution within deeper crustal structure over large parts of the world not yet being known with a sufficient resolution and accuracy, there are several global datasets, which provide more detailed information on the geometry (and density distribution) within shallower crustal structures. Chen and Tenzer (2014) used such datasets for a compilation of the Earth's Spectral Crustal Model (ESCM180) by incorporating more detailed information on the topography, bathymetry, polar ice sheets and geoid surface into the CRUST1.0 model.

In this study, we use the ESCM180 parameters to estimate some fundamental density parameters of the continental and oceanic crustal structures, which are required in the gravimetric forward and inverse modelling of the lithosphere structure including the Moho interface. We use the ESCM180 coefficients to compute the gravity field quantities of major known crustal density structures. The ESCM180 is briefly described in Sect. 2. The 
method of computing the gravity field quantities is reviewed in Sect. 3. The results are presented in Sect. 4. The estimated average densities of major crustal structures are given and compared with previous studies in Sect. 5. We further analyse spatial and spectral characteristics of the corrected gravity fields and their spectral correlation with the crustal geometry. We demonstrate that the signature of crustal density structure prevails at the long-to-medium gravity spectrum and discusses some theoretical and practical consequences of this finding in context of the gravimetric Moho determination (Sect. 6). Here, we also discuss the findings related to a spectral behaviour of the Moho signature in gravity data. Major results and findings are summarized and concluded in Sect. 7.

\section{Earth's Spectral Crustal Model 180}

The ESCM180 consists of 9 individual crustal layers, which describe the geometry and density (or density contrast) distribution within the topography, ocean, polar ice sheets, sediments (3 layers) and consolidated crust (3 layers). The ESCM180 was derived based on refining the CRUST1.0 global crustal model by using the ETOPO1 topographic/bathymetric model (Amante and Eakins 2009) and the DTM2006.0 ice-thickness dataset (Pavlis et al. 2007). The GOCO-03S global geoid model (Mayer-Guerr et al. 2012) was used to define more accurately the geometry of the lower topographic bound (on land) and the upper bathymetric bound (over oceans) instead of assuming only a spherical approximation. Moreover, the depth-dependent seawater density model (Tenzer et al. 2012c) was facilitated in the definition of the ocean density contrast (instead of a uniform seawater density model). The ESCM180 is defined by a set of the spherical upper-bound and lower-bound coefficients $U_{n, m}^{(k+1+i)}$ and $L_{n, m}^{(k+1+i)}$, which combine the information on the geometry and 3-D density (or density contrast) distribution within each individual crustal component. The ESCM180 topographic, bathymetric, ice, sediment and consolidated crust coefficients were generated with a spherical resolution complete to degree 180 of spherical harmonics and up to the third-order terms of a binomial expansion, i.e. $\left\{L_{n, m}^{(k+1)}, U_{n, m}^{(k+1)}: \quad k=0,1,2 ; \quad n, m=0,1, \ldots, 180\right\}$. For the depth-dependent seawater density model, the ESCM180 bathymetric coefficients were extended for additional coefficients up to the second-order (seawater) density terms, i.e. $\left\{L_{n, m}^{(k+1+i)}, U_{n, m}^{(k+1+i)}: \quad k=0,1, \ldots ; \quad i=1,2 ; \quad n, m=0,1, \ldots, 180\right\}$. These coefficients were generated from discrete data according to the following expressions for a spherical harmonic analysis (Tenzer et al. 2012a)

$$
L_{n}^{(k+1+i)}(\Omega)=\left\{\begin{array}{l}
\frac{2 n+1}{4 \pi} \iint_{\Phi} \rho\left(D_{U}, \Omega^{\prime}\right) D_{L}^{k+1}\left(\Omega^{\prime}\right) P_{n}(\cos \psi) \mathrm{d} \Omega^{\prime} \\
=\sum_{m=-n}^{n} L_{n, m}^{(k+1)} Y_{n, m}(\Omega) \quad i=0 \\
\frac{2 n+1}{4 \pi} \iint_{\Phi} \beta\left(\Omega^{\prime}\right) a_{i}\left(\Omega^{\prime}\right) D_{L}^{k+1+i}\left(\Omega^{\prime}\right) P_{n}(\cos \psi) \mathrm{d} \Omega^{\prime} \\
=\sum_{m=-n}^{n} L_{n, m}^{(k+1+i)} Y_{n, m}(\Omega) \quad i=1,2, \ldots I
\end{array}\right.
$$

and 


$$
U_{n}^{(k+1+i)}(\Omega)=\left\{\begin{array}{l}
\frac{2 n+1}{4 \pi} \iint_{\Phi} \rho\left(D_{U}, \Omega^{\prime}\right) D_{U}^{k+1}\left(\Omega^{\prime}\right) P_{n}(\cos \psi) \mathrm{d} \Omega^{\prime} \\
=\sum_{m=-n}^{n} U_{n, m}^{(k+1)} Y_{n, m} \Omega^{\prime}(\Omega) \quad i=0 \\
\frac{2 n+1}{4 \pi} \iint_{\Phi} \beta\left(\Omega^{\prime}\right) a_{i}\left(\Omega^{\prime}\right) D_{U}^{k+1+i}\left(\Omega^{\prime}\right) P_{n}(\cos \psi) \mathrm{d} \Omega^{\prime} \\
=\sum_{m=-n}^{n} U_{n, m}^{(k+1+i)} Y_{n, m}(\Omega) \quad i=1,2, \ldots, I
\end{array}\right.
$$

where $Y_{n, m}$ are the (fully normalized) surface spherical harmonic functions of degree $n$ and order $m, P_{n}$ are the Legendre polynomials for the argument of cosine of the spherical distance $\psi$ between two points $(r, \Omega)$ and $\left(r^{\prime}, \Omega^{\prime}\right)$. The 3-D position is defined in the spherical coordinate system $(r, \Omega)$, where $r$ is the spherical radius and $\Omega=(\phi, \lambda)$ is a spherical direction with the spherical latitude $\phi$ and longitude $\lambda$. The infinitesimal surface element on the unit sphere is denoted as $\mathrm{d} \Omega^{\prime}=\cos \phi^{\prime} \mathrm{d} \phi^{\prime} \mathrm{d} \lambda^{\prime}$, and $\Phi=$ $\left\{\Omega^{\prime}=\left(\phi^{\prime}, \lambda^{\prime}\right): \phi^{\prime} \in[-\pi / 2, \pi / 2] \wedge \lambda^{\prime} \in[0,2 \pi)\right\}$ is the full spatial angle. The integral convolutions in Eqs. (1) and (2) utilize a 3-D density distribution $\rho$ defined by the following regression function

$$
\rho(r, \Omega)=\rho\left(D_{\mathrm{U}}, \Omega\right)+\beta(\Omega) \sum_{i=1}^{I} a_{i}(\Omega)(R-r)^{i} \quad \text { for } R-D_{\mathrm{U}}(\Omega) \geq r>R-D_{\mathrm{L}}(\Omega),
$$

where $\rho\left(D_{\mathrm{U}}, \Omega\right)$ is a (nominal) value of the lateral density at a location $\Omega$ and at a depth $D_{U}$. The 3-D density contrast model with respect to the reference crustal density $\rho^{\text {ref }}$ is defined as

$$
\Delta \rho\left(r, \Omega^{\prime}\right)=\rho^{\mathrm{ref}}-\rho(r, \Omega),
$$

with $\rho(r, \Omega)$ given in Eq. (3). The depth-dependent seawater density model in Eq. (3) was defined for the surface seawater density of $1,027.91 \mathrm{~kg} / \mathrm{m}^{3}$ (cf. Gladkikh and Tenzer 2012) and the parameters (up to the second-order density terms): $\beta=0.00637 \mathrm{~kg} / \mathrm{m}^{3}, a_{1}=$ $0.7595 \mathrm{~m}^{-1}$ and $a_{2}=-4.3984 \times 10^{-6} \mathrm{~m}^{-2}$ (cf. Tenzer et al. 2012d). The adopted density of glacial ice is $917 \mathrm{~kg} / \mathrm{m}^{3}$ (cf. Cutnell and Kenneth 1995). The CRUST1.0 lateral density models were used to represent the density within the sediment and consolidated crustal layers.

\section{ESCM180 Gravity Field}

The ESCM180 coefficients were used to compute the topographic and crust density contrasts (stripping) gravity corrections. We note here that the procedure of removing the gravitational signal of anomalous density structures is known in the literature as a stripping (e.g., Hammer 1963). These gravity corrections were then applied to the gravity disturbance $\delta g$ according to the following scheme

$$
\delta g^{\mathrm{cs}}=\delta g-g^{t}-g^{b}-g^{i}-g^{s}-g^{c},
$$

where $\delta g^{\text {cs }}$ is the consolidated crust-stripped gravity disturbance (Tenzer et al. 2009a); $g^{t}$ is the topographic gravity correction; and $g^{b}, g^{i}, g^{s}$ and $g^{c}$ are, respectively, the stripping gravity corrections due to density contrasts of the ocean (bathymetry), ice, sediment and 
consolidated crust. The GOCO-03S coefficients (Mayer-Guerr et al. 2012) and the parameters of the GRS-80 normal gravity field (Moritz 2000) were used to compute the gravity disturbance $\delta g$ as follows (e.g., Heiskanen and Moritz 1967)

$$
\delta g(r, \Omega)=\frac{\mathrm{GM}}{R^{2}} \sum_{n=0}^{\bar{n}} \sum_{m=-n}^{n}\left(\frac{R}{r}\right)^{n+2}(n+1) T_{n, m} Y_{n, m}(\Omega),
$$

where $\mathrm{GM}=3,986,005 \times 10^{8} \mathrm{~m}^{3} / \mathrm{s}^{2}$ is the geocentric gravitational constant (i.e. the product of Newton's gravitational constant and the total mass of the Earth including the atmosphere), $R=6,371 \times 10^{3} \mathrm{~m}$ is the Earth's mean radius, $\bar{n}$ is the maximum degree of spherical harmonics, and $T_{n, m}$ are the (fully normalized) numerical coefficients of the disturbing gravity potential $T$ (i.e. the difference between the Earth's and normal gravity potentials).

The computation of the gravity corrections in Eq. (5) from the ESCM180 coefficients $\left\{L_{n, m}^{(k+1+i)}, U_{n, m}^{(k+1+i)}: \quad k=0,1, \ldots ; \quad i=0,1, \ldots\right\}$ was realized according to the following expression for a spherical harmonic synthesis (Tenzer et al. 2012a)

$$
g(r, \Omega)=\frac{\mathrm{GM}}{R^{2}} \sum_{n=0}^{\bar{n}} \sum_{m=-n}^{n}\left(\frac{R}{r}\right)^{n+2}(n+1) V_{n, m} Y_{n, m}(\Omega) .
$$

The (fully normalized) potential coefficients $V_{n, m}$ read

$$
V_{n, m}=\frac{3}{2 n+1} \frac{1}{\bar{\rho}^{\text {Earth }}} \sum_{i=0}^{I}\left(\mathrm{Fl}_{n, m}^{(i)}-\mathrm{Fu}_{n, m}^{(i)}\right),
$$

where $\bar{\rho}^{\text {Earth }}=5,500 \mathrm{~kg} / \mathrm{m}^{3}$ is the Earth's mean density (e.g., Novák 2010), and the numerical coefficients $\left\{\mathrm{Fl}_{n, m}^{(i)}, \mathrm{Fu}_{n, m}^{(i)}: \quad i=0,1, \ldots, I\right\}$ are given by

$$
\mathrm{Fl}_{n, m}^{(i)}=\sum_{k=0}^{n+2}\left(\begin{array}{c}
n+2 \\
k
\end{array}\right) \frac{(-1)^{k}}{k+1+i} \frac{L_{n, m}^{(k+1+i)}}{R^{k+1}}
$$

and

$$
\mathrm{Fu}_{n, m}^{(i)}=\sum_{k=0}^{n+2}\left(\begin{array}{c}
n+2 \\
k
\end{array}\right) \frac{(-1)^{k}}{k+1+i} \frac{U_{n, m}^{(k+1+i)}}{R^{k+1}} .
$$

The topographic gravity correction was computed using the average density of the upper continental crust of $2,670 \mathrm{~kg} / \mathrm{m}^{3}$ (Hinze 2003). For consistency, we used the same density value to define the reference crustal density (Eq. 4) for computing the stripping gravity corrections. It is worth mentioning that, depending on the purpose, density contrasts can be defined for an arbitrary reference (background) density model (such as PREM) or the average crustal density. The choice of different reference crustal density will proportionally change the values of stripping gravity corrections and respective corrected gravity field (i.e. the consolidated crust-stripped gravity disturbances), but will not affect the spatial and spectral characteristics of the corrected gravity field. Therefore, such procedure will not have any impact on the gravity interpretation, and the correlation analysis conducted in this study. 


\section{Results}

The global maps of the topographic and crustal components (stripping) gravity corrections are shown in Fig. 1. The step-wise corrected gravity disturbances are shown in Fig. 2. The statistics of the gravity corrections and respective corrected gravity disturbances are summarized in Tables 1 and 2. The gravity corrections and the corrected gravity disturbances were computed with a spectral resolution complete to degree 180 of spherical harmonics. All computations were realized globally on a $1 \times 1$ arc-deg grid of surface points.

The GOCO-03S gravity disturbances globally vary mostly within $\pm 250 \mathrm{mGal}$ (see Fig. 2a). This small range of gravity disturbances indicates that the Earth's lithosphere is in a relatively good isostatic balance at long-to-medium wavelengths. Higher frequency gravity features are seen in mountains with positive gravity disturbances at high elevations as well as over oceanic subduction zones (alongside the uplifted lithosphere). In contrast, large negative values, typically seen along continental sedimentary basins and oceanic subduction zones (alongside the subducted lithosphere), have more pronounced mediumwavelength spatial features. More detailed analysis and interpretation of the gravity field can be found, for instance, in studies of Le Pichon and Talwani (1969), Talwani et al. (1969), Talwani (1970), Kaula (1969, 1972), Lambeck (1971), Dixon et al. (1983), Wessel and Watts (1988), Baudry and Calmant (1991), Jung and Vogt (1992), Smith (1993), Artemjev et al. (1994), Cazenave et al. (1996), Sandwell and Smith (1997), Wang et al. (2011). The application of the topographic gravity correction (Fig. 1a) exhibited the isostatic compensation under orogens, marked by large negative gravity disturbances (see Fig. 2b). The application of the bathymetric gravity correction (Fig. 1b) significantly changed a spatial pattern of marine gravity disturbances, revealing the signature of the ocean-floor relief (see Fig. 2c). The application of the ice gravity correction (Fig. 1c) changed significantly the gravity signal in Greenland and Antarctica in regions with the largest continental ice sheets (see Fig. 2d). This correction exhibited more realistically a possible signature of isostasy in gravity data over these regions; we note that the isostatic signature was to a large extent magnified after applying the topographic gravity correction (calculated for the topographic density of $2670 \mathrm{~kg} / \mathrm{m}^{3}$ ). The application of the sediment gravity correction (Fig. 1d) slightly changed a spatial pattern of the gravity disturbances over large continental sedimentary basins and to some extent enhanced the contrast between the continental and oceanic crustal structures along continental margins, especially over the largest sedimentary accumulations of deep-sea fans (i.e. large marine sediment accumulations deposited on the slope and adjacent sea floor originated during ice-age climatic episodes) and due to the discharge of large rivers (see Fig. 2e). These changes are, however, not as significant as the corresponding changes in gravity maps seen after applying the topographic, bathymetric and ice gravity corrections, because values of the sediment gravity correction are typically less than $100 \mathrm{mGal}$ (cf. Table 1). The consolidated cruststripped gravity disturbances (obtained after applying the final gravity correction due to remaining crustal density heterogeneities; see Fig. 1e) are mostly positive over oceans while negative over continents (see Fig. 2f). The gravity maxima correspond with locations of the old oceanic lithosphere, and the gravity maxima are over large orogens. It is worth mentioning that the atmospheric gravity correction was not applied in this study. Tenzer et al. (2009b) demonstrated that this correction globally varies between -0.18 and $0.03 \mathrm{mGal}$, and it is thus completely negligible (when compared with the applied gravity corrections; see Table 1). 

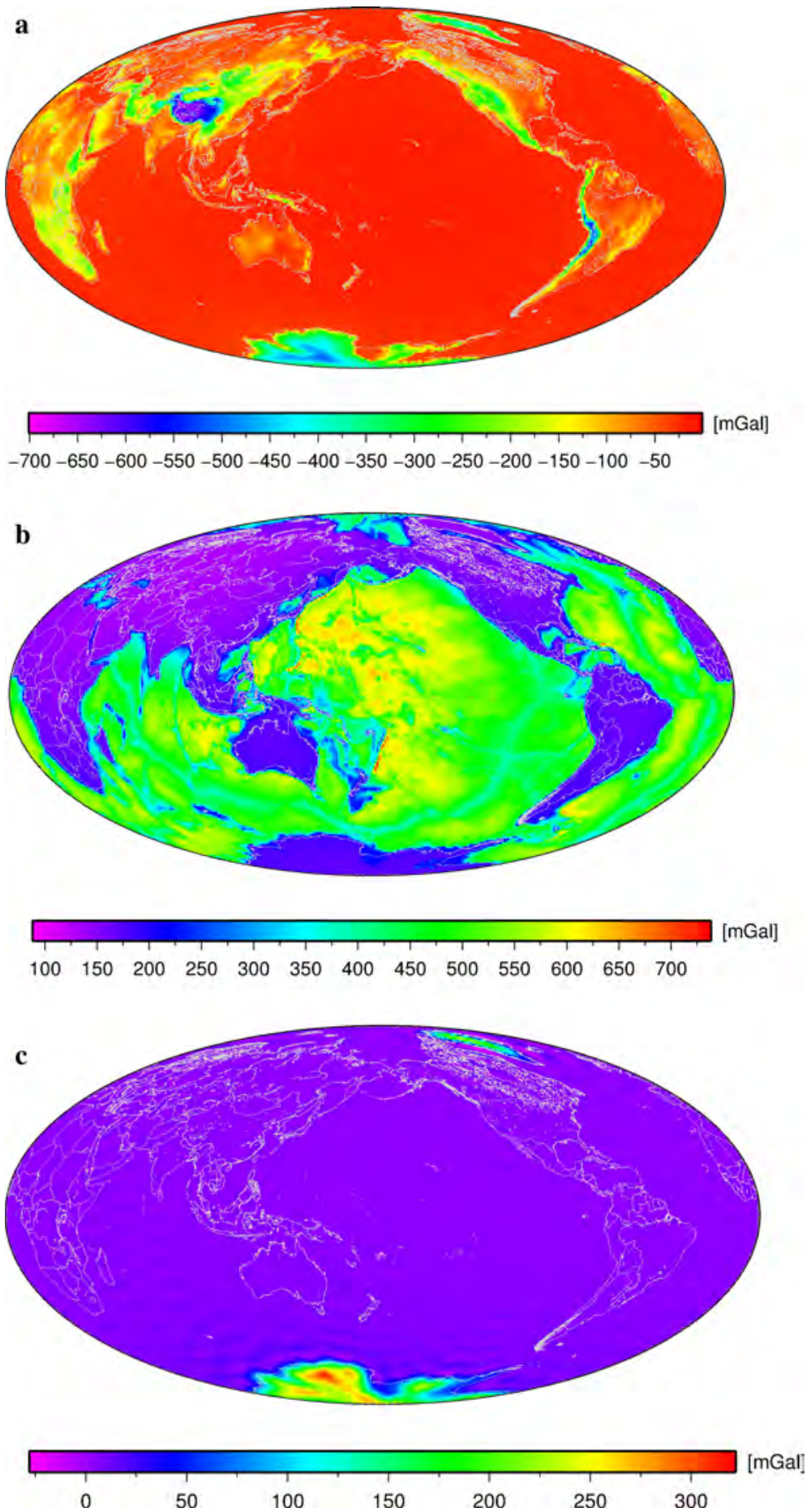
Fig. 1 Global maps of the gravity corrections computed globally on a $1 \times 1$ arc-deg grid of surface points with a spectral resolution complete to degree 180 of spherical harmonics: a the topographic correction $g^{\mathrm{T}}$, $\mathbf{b}$ the bathymetric stripping correction $g^{\mathrm{B}}, \mathbf{c}$ the ice striping correction $g^{\mathrm{I}}, \mathbf{d}$ the sediment stripping correction $g^{\mathrm{S}}$, and e the consolidated crust stripping correction $g^{\mathrm{C}}$
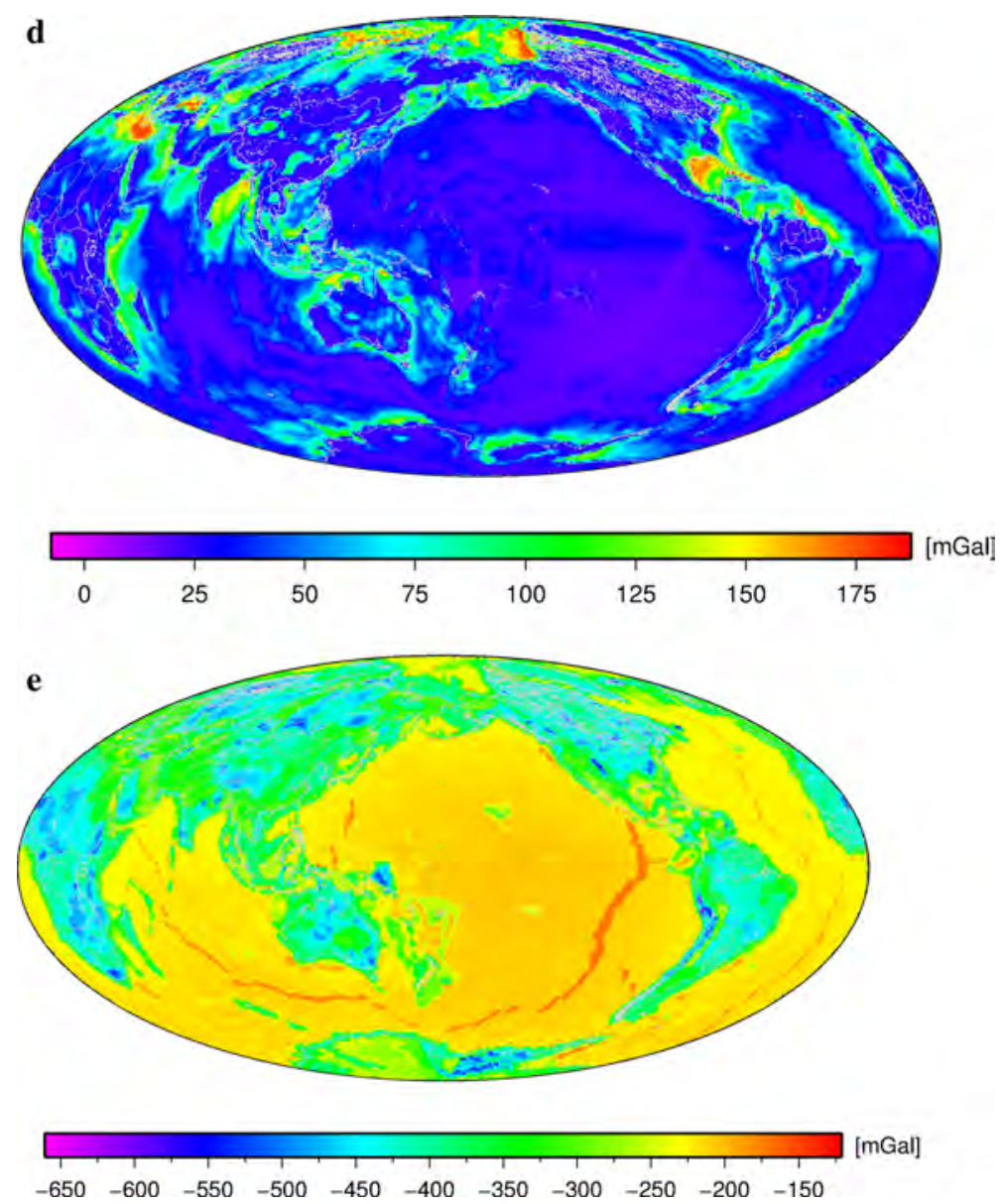

Fig. 1 continued

\section{Analysis}

We used the ESCM180 parameters and respective gravity field quantities for the analysis of average densities of major crustal structures and for the investigation of spatial and spectral characteristics of the consolidated crust-stripped gravity disturbances and their correlation with the crustal geometry.

\subsection{Average Densities of Crustal Structures}

Our analysis of the ESCM180 model revealed that the average crustal density including the seawater is $2,490 \mathrm{~kg} / \mathrm{m}^{3}$, while the density of the solid crust is $2,830 \mathrm{~kg} / \mathrm{m}^{3}$. These 

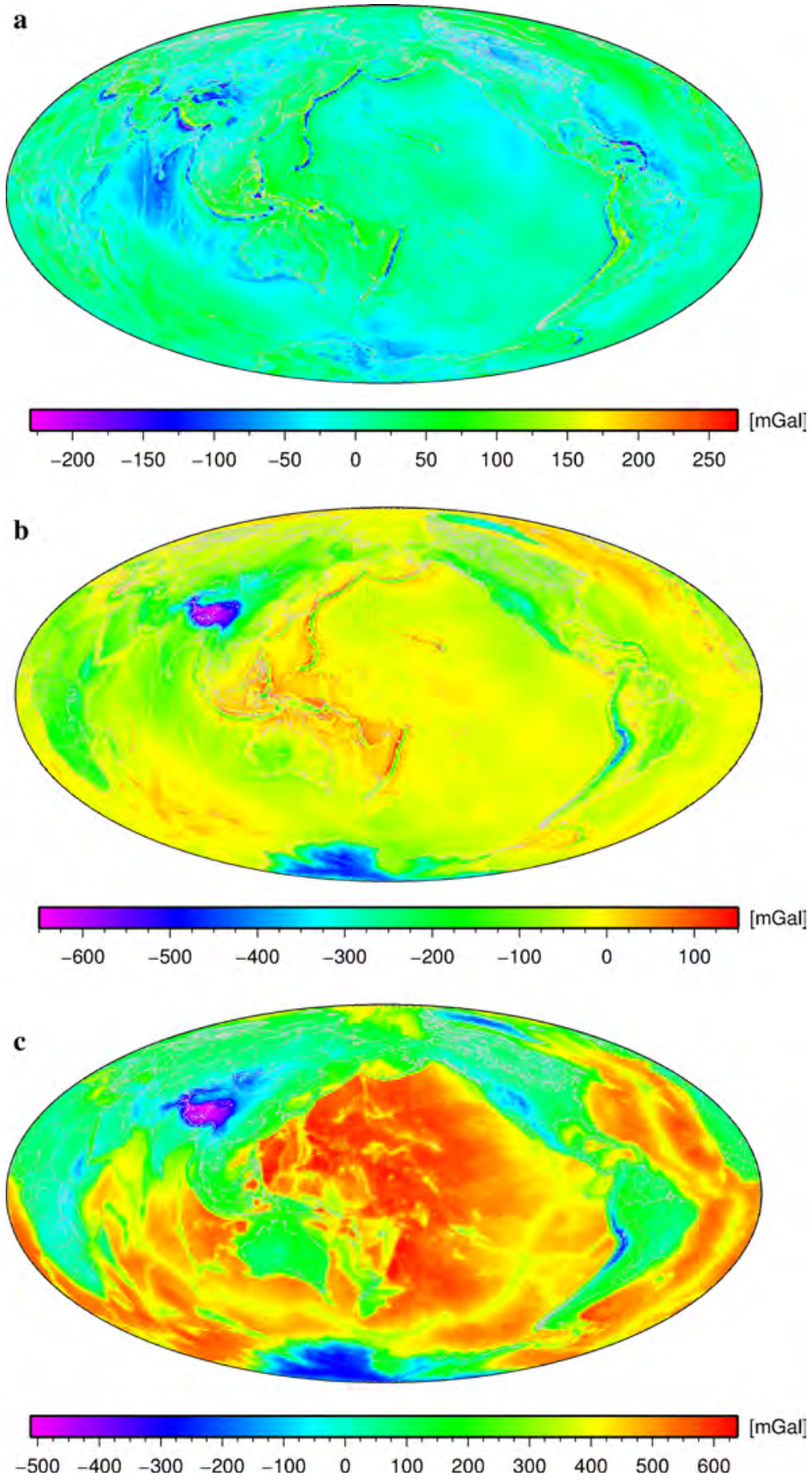
Fig. 2 Global maps of the step-wise corrected gravity disturbances computed globally on a $1 \times 1$ arc-deg grid of surface points with a spectral resolution complete to degree 180 of spherical harmonics: a the GOCE03S gravity disturbances $\delta g, \mathbf{b}$ the topography-corrected gravity disturbances $\delta g^{\mathrm{T}}, \mathbf{c}$ the topography-corrected and bathymetry-stripped gravity disturbances $\delta g^{\mathrm{TB}}, \mathbf{d}$ the topography-corrected and bathymetry- and ice-stripped gravity disturbances $\delta g^{\mathrm{TBI}}$, e the topography-corrected and bathymetry- and ice- and sediments-stripped gravity disturbances $\delta g^{\text {TBIS }}$ and $\mathbf{f}$ the consolidated crust-stripped gravity disturbances $\delta g^{\mathrm{cs}}$

estimates were obtained by applying a spatial average operator over crustal layers (and taking into consideration the convergence of meridians). We also found that the average continental crustal density including continental shelves, consisting of igneous, sedimentary and metamorphic rocks, is $2,790 \mathrm{~kg} / \mathrm{m}^{3}$. Our estimated value of the continental crustal density of $2,790 \mathrm{~kg} / \mathrm{m}^{3}$ differs about $1.6 \%$ from the value of $2,835 \mathrm{~kg} / \mathrm{m}^{3}$ reported by Christensen and Mooney (1995). These two density estimates of the whole continental crust are obviously larger than the average density of only the upper continental crust of $2,670 \mathrm{~kg} / \mathrm{m}^{3}$ (mentioned by Hinze 2003) due to the increasing density within deeper crustal structures.

The oceanic crust, composed primarily of mafic rocks, is typically heavier than the continental crust (e.g., Rogers et al. 2008). Our analysis revealed that the average density of the ESCM180 oceanic crust (without the seawater, but including marine sediments) is $2,860 \mathrm{~kg} / \mathrm{m}^{3}$. This value is smaller than the average density of the oceanic crust (without marine sediments) of $2,890 \mathrm{~kg} / \mathrm{m}^{3}$ estimated by Carlson and Raskin (1984), because the density of marine sediments was not taken into consideration in their analysis. They used seismic refraction data in combination with drilling results, laboratory studies of seismic properties of oceanic and ophiolitic rocks and ophiolite lithostratigraphy. Tenzer and Gladkikh (2014) confirmed a similar value of the average density of the oceanic crust (without marine sediments) of $2,900 \mathrm{~kg} / \mathrm{m}^{3}$ based on the analysis of global samples of marine bedrock densities. These density samples were collected from drilling sites of the Deep Sea Drilling Project (DSDP) and recorded in the National Geophysical Data Center (NGDC) of the U.S. National Oceanic and Atmospheric Administration (NOAA). They also found that the average density of the DSDP marine sediments data is $1,700 \mathrm{~kg} / \mathrm{m}^{3}$. Estimates of the average sediment density can also be found, for instance, in Sclater et al. (1971, 1977, 1985), Crough (1983), Renkin and Sclater (1988), Hayes (1988), Kane and Hayes (1992) and Coffin (1992). These authors provided the average density estimates between 1,700 and $1,950 \mathrm{~kg} / \mathrm{m}^{3}$; for summary, see Sykes (1996).

The comparison of the CRUST2.0 and CRUST1.0 models reveals some substantial differences in density structure within sediments, consolidated crust and upper mantle. The effect of these changes on the respective gravity field is discussed in Sect. 6. These changes affected also the Moho density contrast. The CRUST2.0 Moho density contrast has a small range of values of only $201-451 \mathrm{~kg} / \mathrm{m}^{3}$ (with a mean of $321 \mathrm{~kg} / \mathrm{m}^{3}$ and a standard deviation of $58 \mathrm{~kg} / \mathrm{m}^{3}$ ). The CRUST1.0 Moho density contrast (shown in Fig. 3) is between -40 and $610 \mathrm{~kg} / \mathrm{m}^{3}$. The minima of the CRUST1.0 Moho density contrast correspond with the divergent tectonic plate boundaries, marking distinctively mid-oceanic ridges and continental rift zones (i.e. the West-Antarctic and East-African rift zones). The corresponding density contrast maxima are mainly located under orogens of the Himalaya, Tibet Plateau and Andes.

\subsection{Correlation Analysis of the ESCM180 Gravity Field}

As seen in the global gravity maps (in Fig. 2), successive application of the gravity corrections reveals a gravitational signature, which spatially closely resembles major 

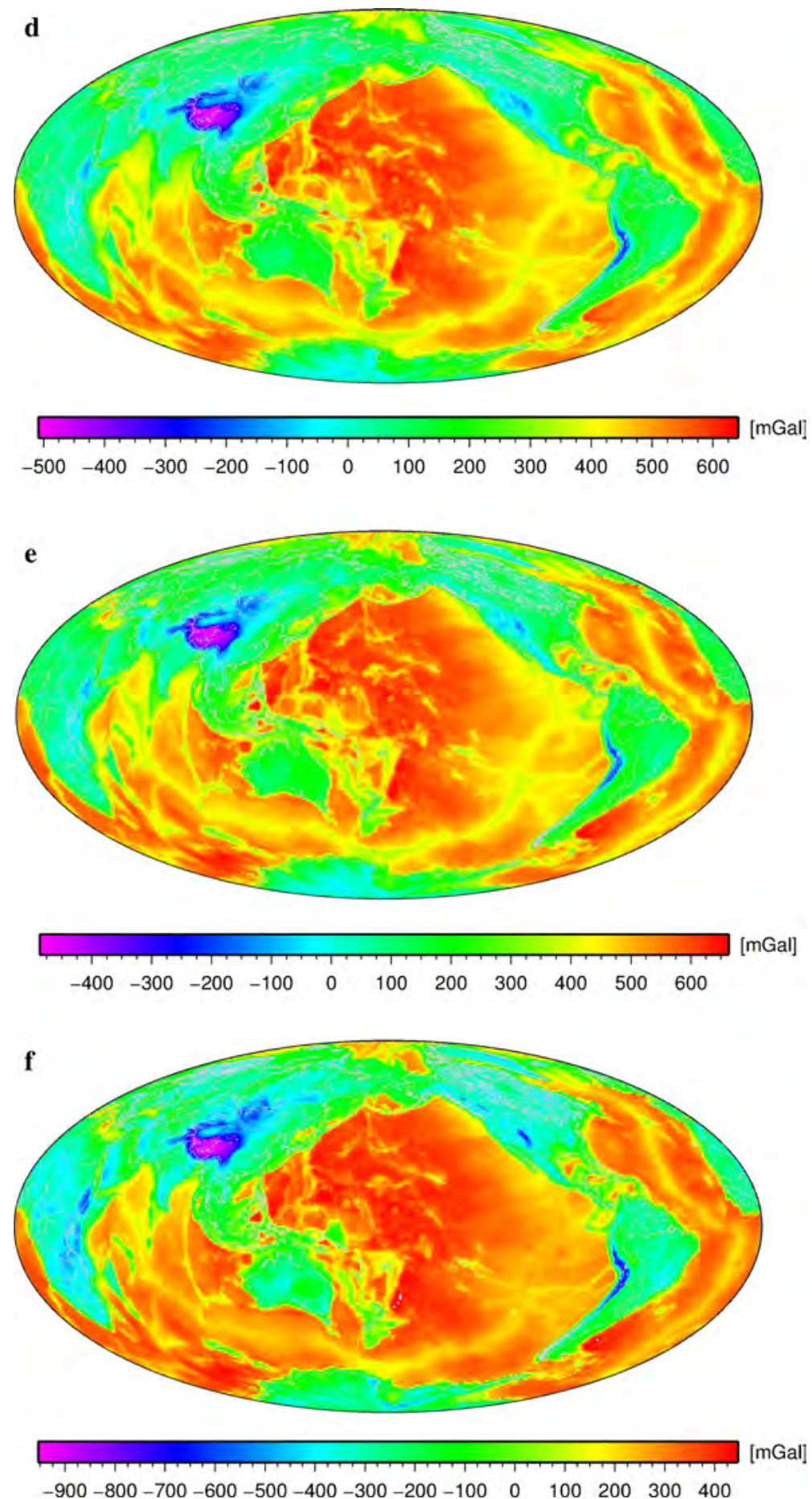

Fig. 2 continued 
Table 1 Statistics of the gravity corrections computed globally on a $1 \times 1$ arc-deg grid of surface points with a spectral resolution complete to degree 180 of spherical harmonics

\begin{tabular}{lcccc}
\hline Gravity corrections & Min $(\mathrm{mGal})$ & Max $(\mathrm{mGal})$ & Mean $(\mathrm{mGal})$ & STD $(\mathrm{mGal})$ \\
\hline$g^{\mathrm{T}}$ & -701 & 5 & -71 & 104 \\
$g^{\mathrm{B}}$ & 89 & 725 & 332 & 165 \\
$g^{\mathrm{I}}$ & -27 & 311 & 25 & 61 \\
$g^{\mathrm{uS}}$ & -3 & 105 & 34 & 21 \\
$g^{\mathrm{mS}}$ & -17 & 87 & 11 & 14 \\
$g^{\mathrm{IS}}$ & -5 & 29 & 1 & 2 \\
$g^{\mathrm{uC}}$ & -141 & 52 & -20 & 24 \\
$g^{\mathrm{mC}}$ & -199 & -22 & -71 & 35 \\
$g^{\mathrm{IC}}$ & -526 & -106 & -202 & 40
\end{tabular}

$g^{\mathrm{T}}$, the topographic correction; $g^{\mathrm{B}}$, the bathymetric stripping correction; $g^{\mathrm{I}}$, the ice striping correction; $g^{\mathrm{uS}}$, $g^{\mathrm{mS}}$ and $g^{\mathrm{IS}}$, the upper-, middle- and lower-sediment stripping corrections; $g^{\mathrm{uC}}, g^{\mathrm{mC}}$ and $g^{\mathrm{lC}}$, the upper, middle and lower consolidated crust stripping corrections

features of the upper and lower crustal geometry. To investigate the correlation between the corrected gravity disturbances and the crustal geometry, we computed the solid topography (i.e. topographic heights on land and bathymetric depths over oceans) on a $1 \times 1$ arc-deg grid from the ESCM180 topographic/bathymetric coefficients (descaled for the density model) up to degree/order of 180. The $1 \times 1$ arc-deg Moho depths were obtained from the CRUST1.0 database. The ESCM180 solid topography and the CRUST1.0 Moho depths are shown in Figs. 4 and 5, respectively. The maximum topographic height reaches $5.5 \mathrm{~km}$, and the maximum bathymetric depth is $7.9 \mathrm{~km}$. The CRUST1.0 Moho depths vary between 7.4 and $74.8 \mathrm{~km}$. The spatial correlation of the stepwise corrected gravity disturbances with the solid topography and the Moho geometry is given by means of Pearson's (1895) linear correlation coefficients in Table 3.

The GOCO-03S gravity disturbances have almost a zero correlation with the solid topography ( -0.01$)$, and their correlation with the Moho geometry is only -0.09 . Interestingly, the Earth's gravity field has a slightly higher (absolute) correlation with the Moho geometry than with the solid topography. This indicates that at the investigated gravity spectrum (up to degree 180 of spherical harmonics), the gravity signatures of crustal and mantle density heterogeneities are more pronounced in the Moho geometry. The higher frequency spectrum of the Earth's gravity field is, on the other hand, dominated by the pattern of the terrain and ocean-floor relief. The application of the topographic and bathymetric gravity corrections significantly increased the (absolute) correlation of the gravity disturbances with the solid topography $(-0.89)$ as well as with the Moho geometry $(-0.95)$. Application of the additional ice, sediment and consolidated crust gravity corrections further increased the (absolute) correlation of the gravity disturbances to -0.95 (with the solid topography) and -0.98 (with the Moho geometry). The correlation of the consolidated crust-stripped gravity disturbances with the CRUST1.0 Moho geometry (of -0.98 ) found in this study agrees very well with the value reported by Tenzer et al. (Tenzer et al. 2012c, see also 2009c). They estimated that the consolidated crust-stripped gravity disturbances have a correlation with the CRUST2.0 Moho geometry of -0.96 . These gravity disturbances thus comprise a maximum gravity signature of the Moho geometry. However, these gravity disturbances contain also the gravity signal of unmodelled mantle heterogeneities (including the core-mantle boundary zone; cf. Peltier 2007) and the 
ESCM180 crustal model uncertainties. Tenzer et al. (2012a, b) estimated that relative errors in these gravity data are about $10 \%$, with most of the errors attributed to uncertainties within the sediment and consolidated crustal layers.

The spatial correlations of the consolidated crust-stripped gravity disturbances with the solid topography and the Moho geometry were investigated individually for the oceanic and continental crustal structures and over orogens. Scatter plots are shown in Fig. 6, and the Pearson's correlation coefficients are summarized in Table 4. As seen in Fig. 6b, d, the isostatic mass balance of the continental crustal structure is typically attributed to a changing depth of compensation (the absolute correlation between the gravity data and the Moho geometry for the continental crust is 0.89), while the isostatic mass balance of the oceanic crust is mainly controlled by a changing density due to a thermal cooling of the oceanic lithosphere (the absolute correlation between the gravity data and the Moho geometry for the oceanic crust is only 0.46). This finding agrees with the fact that the isostatic mass balance over continents (including orogens) is more realistically described by the Airy (1855) model, while the Pratt (1855) model better describes the isostatic mass balance over oceans (e.g., Wild and Heck 2004; Makhloof 2007). Moreover, the gravity data have a very similar systematic trend (with respect to the Moho geometry) over the whole continental crust as well as only over orogens (cf. Fig. 6d, f). Interestingly, the gravity data are also highly spatially correlated with the bathymetry over oceans (Fig. 6a). This correlation with the solid topography decreases over land (Fig. 6c) and become marginal over orogens (Fig. 6e), where the absolute correlation between the gravity data and the topography is only 0.45 . A high spatial correlation of the gravity data with the bathymetry is explained by a thermal contraction of the oceanic lithosphere, which is isostatically compensated by the ocean deepening (e.g., Williams 1975; Parsons and Sclater 1977).

We further investigated the power spectra of the corrected gravity disturbances and their spectral correlation with the crustal geometry. For this purpose, we compared the degree variances and the cumulative degree variances of the (step-wise) corrected gravity disturbances (see Fig. 7). The computation of degree variances was carried out according to Parseval's generalized theorem (van Gelderen and Koop 1997)

$$
\sigma_{n}^{2}(\delta g)=\sum_{m=-n}^{n}\left(T_{n, m}\right)^{2},
$$

where $T_{n, m}$ are the coefficients of the disturbing potential $T$ (see Eq. 2). The corresponding cumulative degree variances were calculated from

$$
\Theta_{N}(\delta g)=\sum_{n=0}^{\bar{n}} \sigma_{n}^{2}(\delta g) .
$$

The degree correlation coefficients (cf. Phillips and Lambeck 1980) were calculated to assess a spectral harmonic correlation between the corrected gravity disturbances and the crustal geometry. For a correlation between the gravity disturbances $\delta g$ and the Moho depths $D$, we have

$$
\theta_{n}^{2}(\delta g, D)=\frac{\sum_{m=-n}^{n}\left(T_{n, m}, D_{n, m}\right)}{\sqrt{\sigma_{n}^{2}(\delta g) \sigma_{n}^{2}(D)}},
$$

where $\sigma_{n}^{2}(D)$ are the degree variances of the Moho-depth spherical coefficients $D_{n, m}$, i.e. 

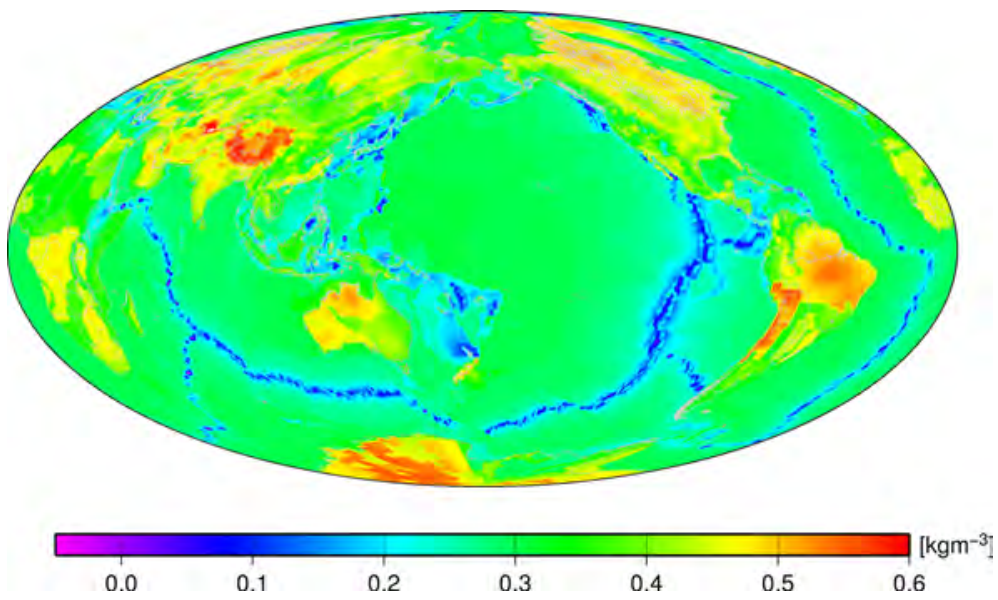

Fig. 3 CRUST1.0 Moho density contrast

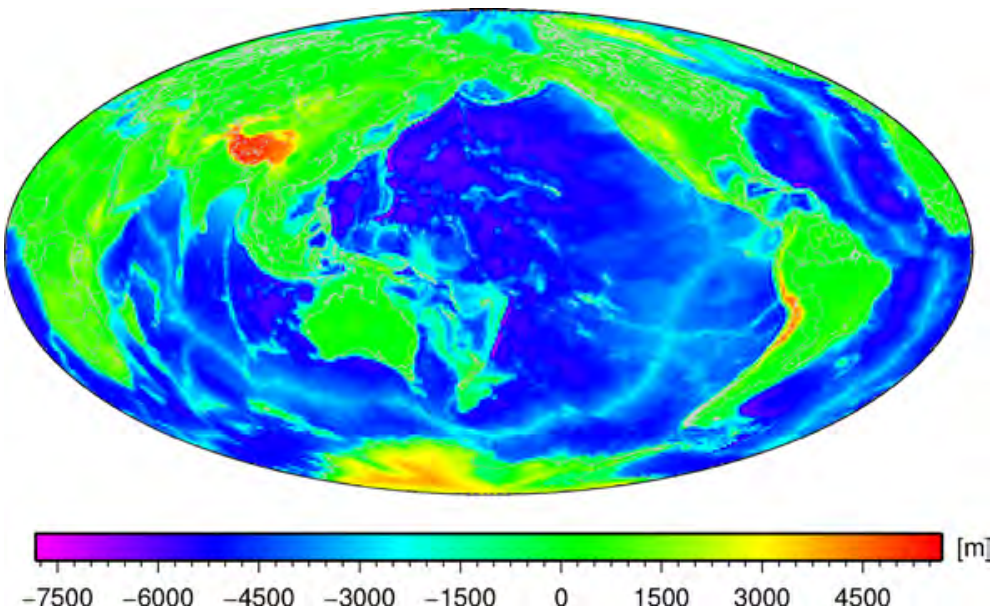

Fig. 4 Solid topography generated on a $1 \times 1$ arc-deg grid with a spectral resolution complete to degree/ order 180

Table 3 Correlations of the step-wise corrected gravity disturbances with the solid topography and the CRUST1.0 Moho geometry

\begin{tabular}{lll}
\hline Gravity disturbances & \multicolumn{2}{l}{ Correlation with } \\
\cline { 2 - 3 } & Solid topography & Moho geometry \\
\hline$\delta g$ & -0.01 & -0.09 \\
$\delta g^{\mathrm{T}}$ & -0.55 & -0.68 \\
$\delta g^{\mathrm{TB}}$ & -0.89 & -0.95 \\
$\delta g^{\mathrm{TBI}}$ & -0.92 & -0.97 \\
$\delta g^{\mathrm{TBIS}}$ & -0.94 & -0.97 \\
$\delta g^{\mathrm{cs}}$ & -0.95 & -0.98 \\
\hline
\end{tabular}




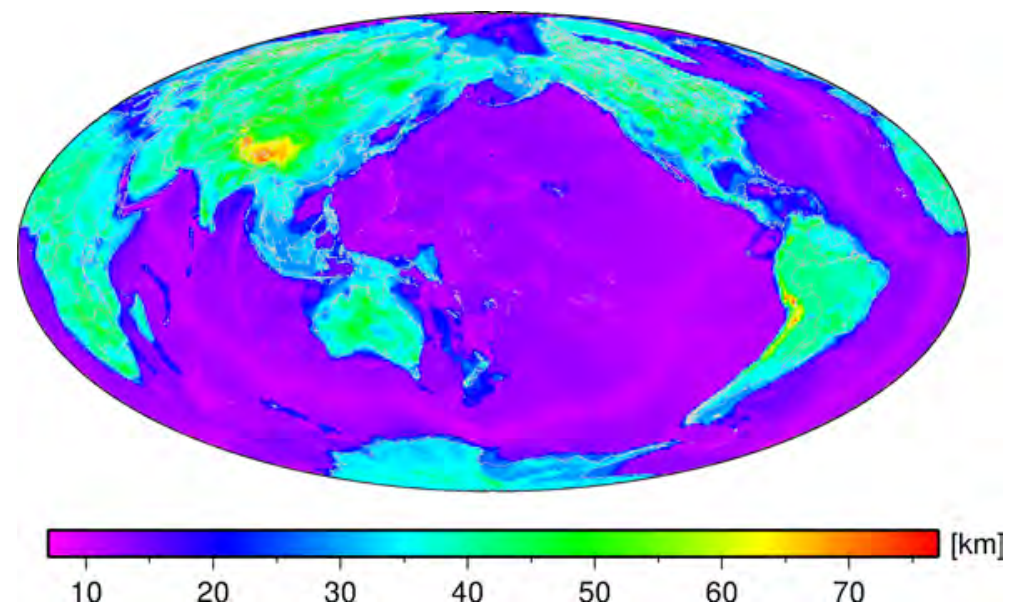

Fig. 5 CRUST1.0 Moho depths

$$
\sigma_{n}^{2}(D)=\sum_{m=-n}^{n}\left(D_{n, m}\right)^{2}
$$

The Moho-depth spherical coefficients $D_{n, m}$ were generated from the discrete values of the CRUST1.0 Moho depths $D$ according to the following expression

$$
\sum_{m=-n}^{n} D_{n, m} Y_{n, m}(\Omega)=\frac{2 n+1}{4 \pi} \iint_{\Phi} D\left(\Omega^{\prime}\right) P_{n}(\cos \psi) \mathrm{d} \Omega^{\prime} .
$$

The same computations were conducted for the solid topography. The spectral correlation of the (step-wise) corrected gravity disturbances with the crustal geometry (up to degree of 180) is shown in Fig. 8.

All investigated types of the gravity disturbances comprise the largest gravity signal at long wavelengths, while a higher frequency contribution almost monotonically attenuates with an increasing degree of spherical harmonics. Except for the ice gravity correction, the application of the gravity corrections increased the degree variances at almost the entire investigated gravity spectrum (up to degree 180). This spectral behaviour corresponds to spatial characteristics of the corrected gravity disturbances of which range of values mostly increased after a subsequent application of each individual gravity correction. The application of the ice gravity correction, on the other hand, substantially reduced (in absolute sense) large negative values of the gravity disturbances in polar areas of Greenland and Antarctica. Consequently, these changes translated into some minor (positive as well as negative) differences in degree variances, especially at the long-wavelength gravity spectrum. The largest changes in degree variances were caused by applying the topographic and bathymetric gravity corrections. The application of the topographic gravity correction changed the gravity spectrum especially at the long-to-medium wavelengths up to degree of $\sim 60$, while changes due to applying the bathymetric gravity correction are seen up to degree of $\sim 100$. Another significant change in the gravity spectrum is due to applying the consolidated crust gravity correction. The differences in degree variances (before and after applying this correction) almost monotonously increased with an increasing 

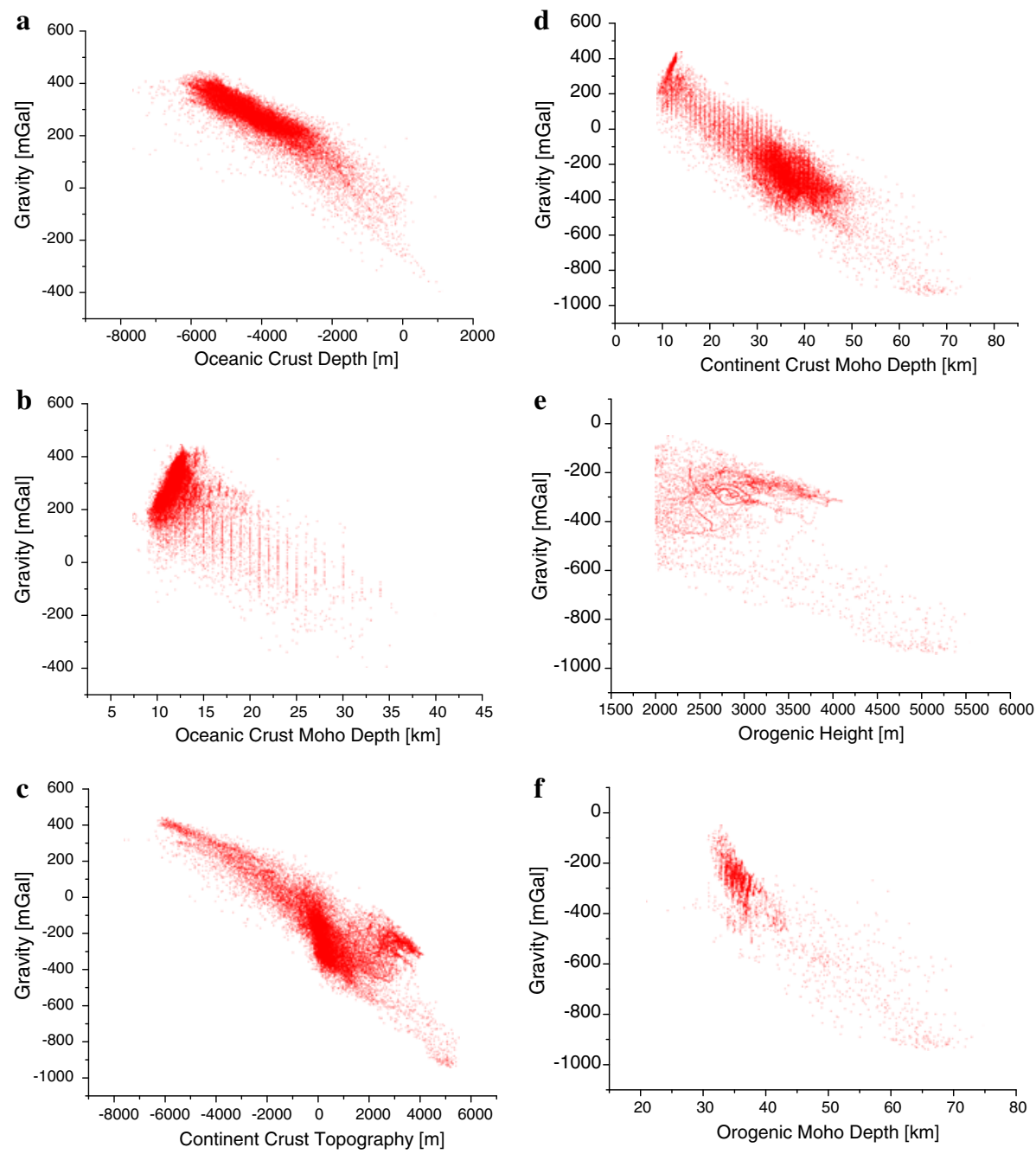

Fig. 6 Scatter plots of the consolidated crust-stripped gravity disturbances versus: a the oceanic crustal bathymetry, $\mathbf{b}$ the Moho geometry of the oceanic crust, $\mathbf{c}$ the continental crustal topography, $\mathbf{d}$ the Moho geometry of the continental crust, $\mathbf{e}$ the orogenic topography and $\mathbf{f}$ the Moho geometry under orogens

Table 4 Correlations of the consolidated crust-stripped gravity disturbances with the solid topography and the CRUST1.0 Moho geometry for the oceanic and continental crustal structures and orogens

\begin{tabular}{llll}
\hline Crustal structure & Topography & Bathymetry & Moho geometry \\
\hline Oceanic crust & - & -0.90 & -0.46 \\
Continent crust & -0.80 & - & -0.89 \\
Orogens & -0.45 & - & -0.89 \\
\hline
\end{tabular}

frequency, while below degree $\sim 10$, these changes are still minor. Small changes in the gravity spectrum attributed to the sediment gravity correction are also recognized, especially at the medium wavelengths above the spherical harmonic degree of $\sim 40$. 

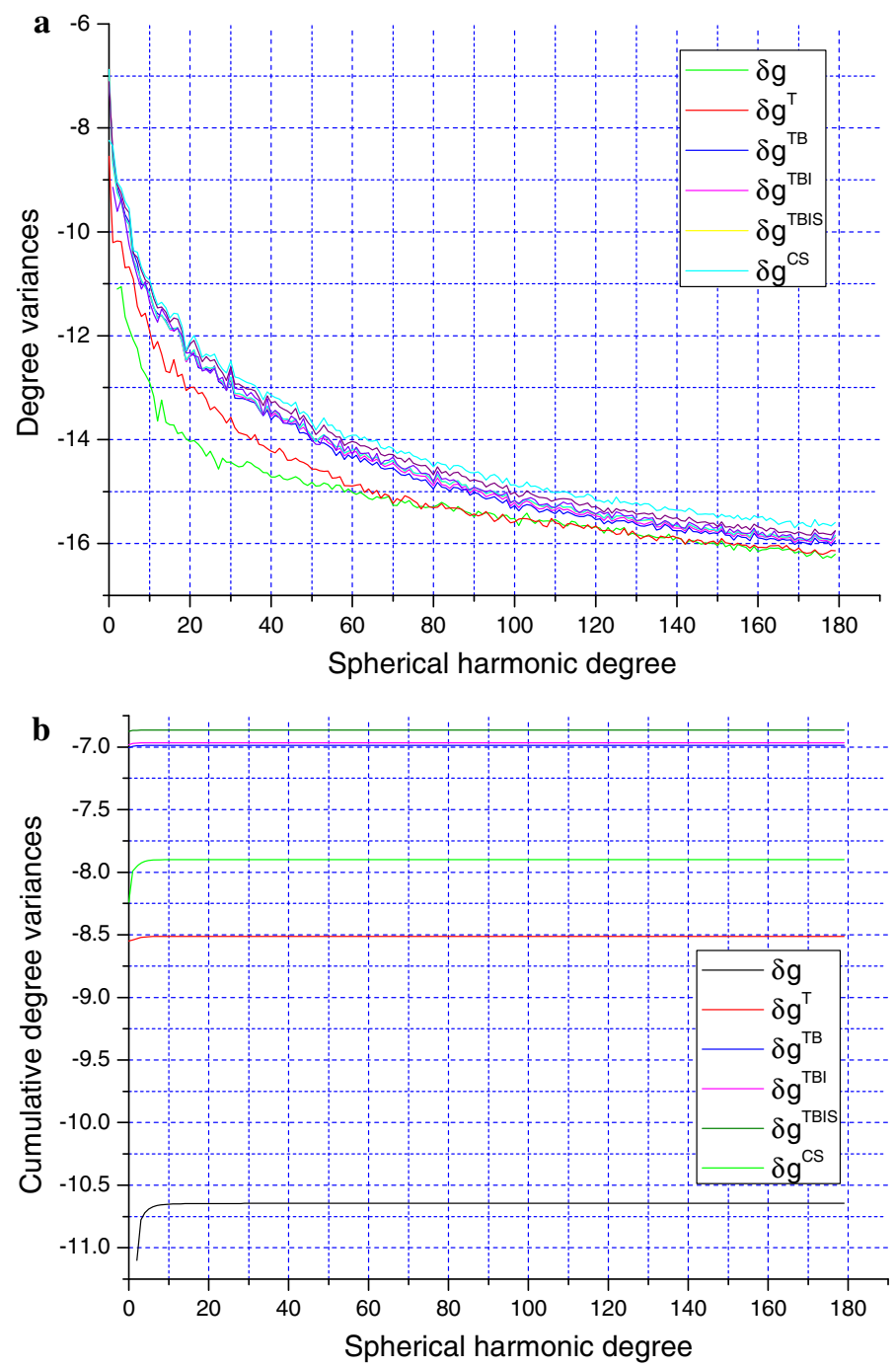

Fig. 7 Power spectrum of the step-wise corrected gravity disturbances: a the degree variances and $\mathbf{b}$ the cumulative degree variances (complete to degree of 180 of the spherical harmonics). Log scale is used for vertical axis

The topography-corrected and bathymetry-stripped gravity disturbances have (among all investigated types of the gravity disturbances) the largest correlation with the solid topography at the whole investigated gravity spectrum. Their largest absolute correlation is at the long wavelengths. This absolute correlation decreases (in absolute sense) to less than 0.6 (at degree 180). The application of the ice gravity correction changed only slightly the correlation pattern at the long wavelengths up to degree of $\sim 45$. The application of the gravity corrections due to anomalous density structures within the sediments and remaining crustal structures further decreased this (absolute) correlation with the solid topography. The largest changes are attributed to crustal density structures, which changed the 

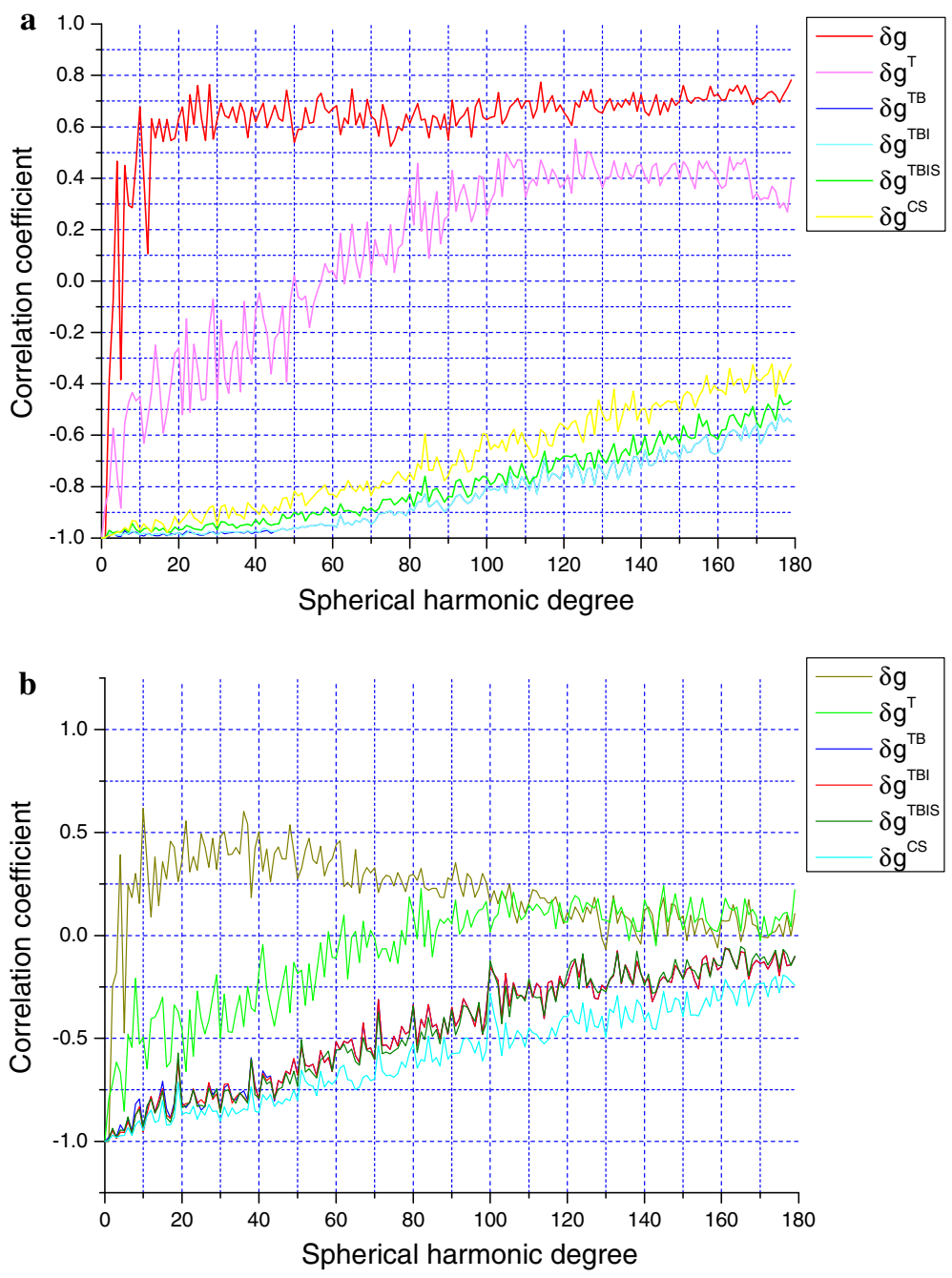

Fig. 8 Correlation spectrum of the step-wise corrected gravity disturbances with: a the solid topography and $\mathbf{b}$ the Moho geometry (complete to degree 180 of spherical harmonics)

correlation pattern especially at higher frequencies beyond degree 80 . The spectral correlation between the topography-corrected gravity disturbances and the solid topography has (absolute) maxima at the long wavelengths, but substantially decreased to $\sim 0.4$ above degree 30 . In addition to this prevailing trend, we also observe large oscillations in this correlation pattern. The GOCO-03S gravity disturbances have (mostly) a negative correlation up to degree 5. Above this degree, the correlation becomes positive and exceeds 0.6 above degree $\sim 20$, where it remains almost unchanged with some minor fluctuations. Both the GOCO-03S and topography-corrected gravity disturbances are thus positively as well as negatively correlated with the solid topography, while the rest of the investigated gravity disturbances have only a negative correlation. 
As seen in Fig. 7b, the signature of the Moho geometry in the Earth's external gravity field is partially recognized at a medium part of the correlation spectrum, approximately at degree 10-80, where the (absolute) spectral correlation exceeds 0.25 and reaches a maximum of $\sim 0.5$. This correlation further attenuates at higher degrees. In contrast, at the long wavelengths, the spectral correlation between the Earth's gravity field and the Moho geometry has large oscillations (with positive as well as negative correlation). The application of the topographic gravity correction changed the spectral correlation with the Moho geometry, especially at the long-to-medium wavelengths up to degree of $\sim 100$. At this interval, we observe mostly a negative correlation with the largest (absolute) values at the long wavelengths, and decreasing trend up to degree of $\sim 60$. Above this degree, the correlation is almost completely absent. Large negative correlation at the long wavelengths is related to large negative values of the topography-corrected gravity disturbances over orogens. The application of the bathymetric gravity correction increased most significantly the (absolute) correlation over the whole investigated gravity spectrum. The correlation with the Moho geometry is everywhere negative with the (absolute) maxima at the long wavelengths, and an attenuating trend (in absolute sense) towards higher degrees, with the (absolute) correlation less than 0.25 above degree of $\sim 100$. The changes in correlation pattern attributed to the sediment and ice gravity corrections are minor. The application of the consolidated crust gravity correction, on the other hand, increased the correlation more significantly at the whole spectrum above degree 10 , with a gradually increasing absolute correlation, especially at the medium and higher part of the gravity spectrum. The consolidated crust-stripped gravity disturbances have the largest correlation with the Moho geometry at the whole gravity spectrum. The maximum (absolute) correlation is at the long wavelengths (more than 0.9 below degree of 20), while this correlation almost monotonously attenuates with an increasing frequency to less than 0.25 (at degree 180).

\section{Discussion}

The comparison of the numerical results in this study (see Tables 1,2 ) with the results presented in Tenzer et al. (2009a) revealed some substantial differences in values of the sediment and consolidated crust gravity corrections and respective corrected gravity disturbances. Tenzer et al. (2009a) reported the range of the sediment gravity correction (computed using the CRUST2.0 soft and hard sediment data) between -7 and $122 \mathrm{mGal}$ (with a mean of $35 \mathrm{mGal}$ and a standard deviation of $22 \mathrm{mGal}$ ). Our updated results have shown that this correction varies between 0 and $185 \mathrm{mGal}$ (with a mean of $45 \mathrm{mGal}$ and a standard deviation of $32 \mathrm{mGal}$ ). Much larger differences were found in values of the consolidated crust gravity correction. For the CRUST2.0, this correction varies between -871 and $-264 \mathrm{mGal}$ (with a mean of $-421 \mathrm{mGal}$ and a standard deviation of $126 \mathrm{mGal}$ ). The CRUST1.0 result is between -661 and $-143 \mathrm{mGal}$ (with a mean of $293 \mathrm{mGal}$ and a standard deviation of $88 \mathrm{mGal}$ ). These large disagreements in the sediment and consolidated crust gravity corrections propagated into large differences between the computed values of the consolidated crust-stripped gravity disturbances. According to updated results, these gravity disturbances vary from -954 to $460 \mathrm{mGal}$ (with a mean of $38 \mathrm{mGal}$ and a standard deviation of $275 \mathrm{mGal}$ ), while the CRUST2.0 solution was between $-1,236$ and $437 \mathrm{mGal}$ (with a mean of $52 \mathrm{mGal}$ and a standard deviation of $301 \mathrm{mGal}$ ). We note here that the facilitation of the latest seismic data considerably improved the accuracy of the CRUST1.0 over certain parts of the world (Laske et al. 2012). Despite the fact that large uncertainties are still expected in sediment and consolidated crustal data, the 
application of respective gravity corrections slightly increased the correlation of the corrected gravity field with the Moho geometry (Sect. 5.2). We thus recommend applying these gravity corrections in the gravimetric Moho modelling.

The application of the gravity corrections due to major crustal density structures changed the gravity spectrum mainly at the long-to-medium wavelengths. This finding has important consequences in the context of mathematical methods applied for a separation of the gravitational signal of deep sources from shallower density structures. Since a global 3 -D density model of the whole mantle is not yet available publically, the direct gravimetric forward modelling of the mantle density heterogeneities is not possible. This unmodelled gravitational signal, however, causes a systematic bias in gravity data, which are used, for instance, in gravimetric Moho modelling. To overcome this problem, some authors proposed and applied various methods of separating the gravity signals attributed to deep and shallower sources, such as a spectral filtering or spectral frequency decomposition. Bagherbandi and Sjöberg (2012), for instance, presented a procedure of treating the long-wavelength gravity signal of the mantle in solving the Vening Meinesz-Moritz (VMM) inverse problem of isostasy (see Sjöberg 2009). They determined a spectral depth of the gravity signal by degree based on Bruns' formula as suggested by Eckhardt (1983) and Bowin et al. (1986). The spherical harmonics that have the spectral depth below a certain limit (chosen, for instance, as the maximum Moho depth) can then be removed from the isostatic gravity field in prior of solving the VMM problem. Our results, however, showed that this procedure is not unique, because the long-wavelength gravity spectrum comprises not only the gravitational signal of mantle heterogeneities but also the gravitational signal of crustal structure. Consequently, this procedure will remove also the signature of the Moho geometry at the long-wavelength gravity spectrum. This is also evident from Fig. 9, where we plotted the degree variances of the CRUST1.0 crustal layers (topography, ocean, polar ice sheets, sediment and consolidated crust) and the Moho geometry. The spectral signal of the crustal layers is pronounced at the long-to-medium wavelengths (see Fig. 9a). Similarly, the Moho geometry is dominated mainly by the longwavelength features over a more detailed structure of the Moho geometry at higher frequencies (see Fig. 9b). This spectral characteristic of the Moho geometry is explained by more localized features, which have the largest horizontal spatial variations of the crustal thickness (e.g., the boundaries between the continental and oceanic lithosphere, the contrast between the crustal thickness under orogens and continental sedimentary basins, large horizontal spatial variations of the crustal thickness under orogens). On the other hand, the long-wavelength features are dominated by a relatively smooth Moho geometry beneath most of the oceanic crust and under the continental crust with large sedimentary accumulations. The isostatic mass balance depends on loading and effective elastic thickness, rigidity, rheology of the lithosphere and viscosity of the asthenosphere (e.g., Watts 2001). Moreover, the glacial isostatic adjustment, present-day glacial melting, plate tectonics and flexure, mantle convection and other geodynamic processes contribute to the overall isostatic balance (e.g., Kaban et al. 2003, 2004). Some of the long-wavelength features in the Moho geometry are likely attributed to these phenomena. Braitenberg et al. (2006) and Wienecke et al. (2007), for instance, demonstrated that the misfit of the isostatic assumption of the Moho interface to a long-wavelength part of the gravity field is explained by large sedimentary basins and rigidity variations of the lithospheric plates.

The spectral correlation analysis revealed that the consolidated crust-stripped gravity disturbances have a maximum correlation with the Moho geometry, especially at the long wavelengths. This (absolute) correlation decreased with an increasing frequency to $\sim 0.25$ (at degree 180). Since the CRUST1.0 model has a $1 \times 1$ arc-deg resolution (which 
corresponds to a spectral resolution up to degree 180), we were not able to confirm that this spectral correlation further decreases beyond degree 180. Nevertheless, we expect that a high-frequency part of the gravity spectrum is not sensitive on a detailed pattern of the Moho geometry, because of a noise in gravity data caused mainly by crustal model uncertainties. The gravimetric determination of a more detailed structure of the Moho geometry is thus possible only if a crustal density model is available with a relatively high resolution and accuracy. Alternatively, gravity and seismic data should be inverted simultaneously. Following this principle, Braitenberg and Zadro (1999), for instance, proposed a method based on the iterative 3-D gravity inversion with integrated seismic data. Sjöberg and Bagherbandi (2011) developed and applied a least-squares approach, which combined seismic and gravity data in the VMM isostatic inverse scheme for a simultaneous estimation of the Moho depth and density contrast. They also presented and applied the non-isostatic correction approach to model systematic discrepancies between isostatic and seismic models (cf. Bagherbandi and Sjöberg 2012).

\section{Summary and Concluding Remarks}

Our results confirmed differences in density structures of the oceanic and continental crust; the average continental crustal density was found to be $2,790 \mathrm{~kg} / \mathrm{m}^{3}$, while the average oceanic crustal density is $2,860 \mathrm{~kg} / \mathrm{m}^{3}$ (when disregarding the seawater). The average density of the whole oceanic crust (including the seawater) is $2,490 \mathrm{~kg} / \mathrm{m}^{3}$. The average crustal density of the whole crust with and without the seawater is 2,490 and $2,830 \mathrm{~kg} / \mathrm{m}^{3}$, respectively.

The updated results of the gravimetric forward modelling based on the CRUST1.0 revealed some substantial changes in the gravity corrections and respective corrected gravity disturbances computed in previous study (cf. Tenzer et al. 2009a). Most of these differences are attributed to sediment and consolidated crustal layers. According to the results presented in this study (cf. Table 2), the consolidated crust-stripped gravity disturbances globally varies from -954 to $460 \mathrm{mGal}$, with a mean of $38 \mathrm{mGal}$ and a standard deviation of $275 \mathrm{mGal}$. Tenzer et al. (2009a) reported the range of these values between $-1,236$ and $437 \mathrm{mGal}$, with a mean of $52 \mathrm{mGal}$ and a standard deviation of $301 \mathrm{mGal}$.

The correlation analysis of the (step-wise) corrected gravity disturbances with the crustal geometry has shown that the consolidated crust-stripped gravity disturbances have a maximum (absolute) correlation with the Moho geometry of 0.98 (cf. Table 3). These gravity data, however, still comprise the (unmodelled) gravitational signal of compositional and thermal structures within the deep mantle (including the core-mantle boundary zone) as well as uncertainties of the ESCM180 crustal density structures (mainly attributed to errors within the CRUST1.0 sediment and consolidated crustal data). The actual Moho geometry is also a result of additional geodynamical processes (such as crustal flexure, glacial isostatic adjustment and tectonics), which cannot be modelled and corrected for directly by applying the gravimetric forward modelling of inner density structures. Consequently, these geodynamical processes cannot be described by an isostatic model based on the adopted hypothesis about the mass balance. The gravitational contributions of these geodynamical processes effects are described by specific functional models (see e.g., Watts 2001; Turcotte and Schubert 2002).

We demonstrated that the application of the topographic and stripping gravity corrections due to major known anomalous crustal density structures revealed a gravity signature of the Moho geometry, especially at the end of the long-to-medium part of the gravity 

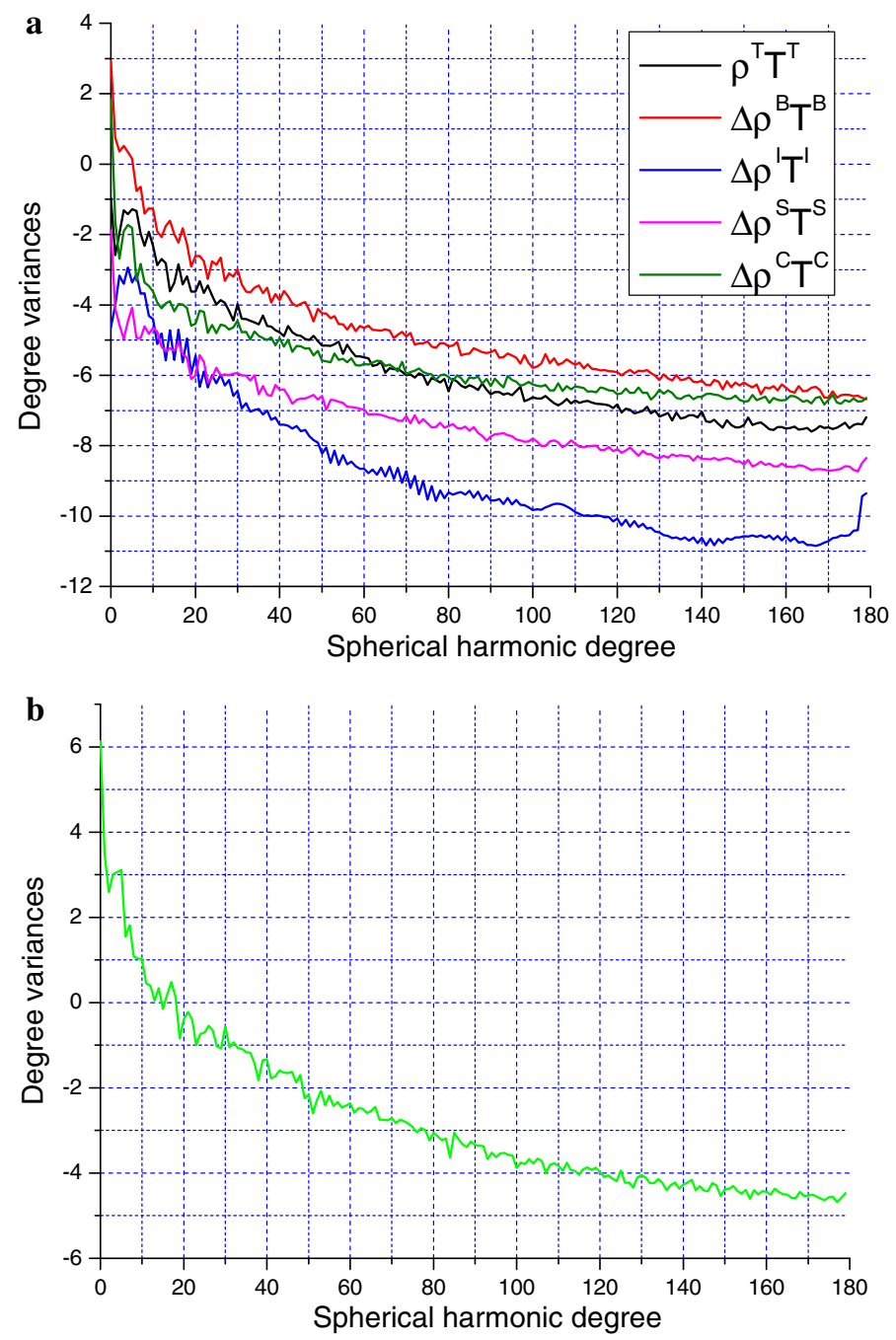

Fig. 9 Power spectrum of: a the ESCM180 crustal density components, and b the CRUST1.0 Moho geometry. The crustal density components were evaluated from the ESCM1 180 coefficients as a product $\rho \mathrm{T}$ or $\Delta \rho \mathrm{T}$; where $\rho$ and $\Delta \rho$ are the density or density contrast, respectively, and $T$ is the thickness of the crustal layers ( $T$ topography, $B$ bathymetry, $I$ ice, $S$ sediment, $C$ consolidated crust). Log scale is used for vertical axis

spectrum. Since the global gravity models are currently available with a relatively high accuracy and resolution, we expect that gravimetric solutions constrained with seismic data should improve the global and regional results of the Moho determination. The correlation of the consolidated crust-stripped gravity disturbances with the Moho geometry at higher frequencies is suppressed by the noise in gravity data due to uncertainties in crustal density models. In local studies, an accurate knowledge of the crustal density structure obtained mainly from seismic surveys) is essential for a gravimetric modelling of more detailed features in the Moho geometry. 
Table 2 Statistics of the step-wise corrected gravity disturbances computed globally on a $1 \times 1$ arc-deg grid of surface points with a spectral resolution complete to degree 180 of spherical harmonics

\begin{tabular}{lllll}
\hline Gravity disturbance & Min $(\mathrm{mGal})$ & Max $(\mathrm{mGal})$ & Mean $(\mathrm{mGal})$ & STD $(\mathrm{mGal})$ \\
\hline$\delta g$ & -229 & 257 & -1 & 30 \\
$\delta g^{\mathrm{T}}$ & -648 & 167 & -72 & 107 \\
$\delta g^{\text {TB }}$ & -511 & 634 & 260 & 233 \\
$\delta g^{\text {TBI }}$ & -508 & 638 & 285 & 202 \\
$\delta g^{\text {TBIuS }}$ & -494 & 664 & 319 & 203 \\
$\delta g^{\text {TBIuSmS }}$ & -487 & 669 & 330 & 200 \\
$\delta g^{\text {TBIS }}$ & -486 & 669 & 331 & 200 \\
$\delta g^{\text {TBISuC }}$ & -579 & 667 & 311 & 219 \\
$\delta g^{\text {TBISuCmC }}$ & -709 & 625 & 240 & 249 \\
$\delta g^{\text {cs }}$ & -954 & 460 & 38 & 275 \\
\hline
\end{tabular}

$\delta g$, the GOCO-03S gravity disturbances, $\delta g^{\mathrm{T}}$ the topography-corrected gravity disturbances; $\delta g^{\mathrm{TB}}$, the topography-corrected and bathymetry-stripped gravity disturbances; $\delta g^{\mathrm{TBI}}$, the topography-corrected and bathymetry- and ice-stripped gravity disturbances; $\delta g^{\mathrm{TBIS}}$, the topography-corrected and bathymetry- and ice- and sediment-stripped gravity disturbances (including intermediate values of $\delta g^{\text {TBIuS }}$-after applying the upper-sediment stripping correction to $\delta g^{\mathrm{TBI}}$ and $\delta g^{\mathrm{TBIuSmS}}$-after applying the upper- and middlesediment stripping corrections to $\left.\delta g^{\mathrm{TBI}}\right) ; \delta g^{\mathrm{cs}}$, and the consolidated crust-stripped gravity disturbances (including the intermediate values of $\delta g^{\mathrm{TBISuC}}$-after applying the upper-crust stripping correction to $\delta g^{\mathrm{TBIS}}$ and $\delta g^{\mathrm{TBISuCmC}}$-after applying the upper- and middle-crust stripping corrections to $\delta g^{\mathrm{TBIS}}$ )

Since a 3-D global mantle density model is not yet available, the direct gravimetric forward modelling of mantle heterogeneities is not possible. As a consequence, alternative methods have been developed and applied in order to remove the unmodelled gravitational signal of the mantle, such as spectral decomposition and spectral filtering techniques; assuming that the gravitational signal of deep mantle structures (including the core-mantle boundary zone) contributes mainly to the long-wavelength gravity spectrum. However, the gravitational signals of the mantle and crust cannot be separated uniquely. As we have shown, the gravitational signature of crustal structures is presented to a large extent at the long-wavelength gravity spectrum. The application of these mathematical techniques will thus remove not only the gravitational signal from the mantle, but eventually also the longwavelength gravitational signature of the Moho geometry. Such methods can then be applied only in regional and local gravimetric studies focusing on a more detailed structure of the Moho geometry, while their application in global studies could introduce systematic errors in the gravimetric Moho solution.

\section{References}

Airy GB (1855) On the computations of the effect of the attraction of the mountain masses as disturbing the apparent astronomical latitude of stations in geodetic surveys. Phil Trans Roy Soc (Lond) B 145:101-104

Amante C, Eakins BW (2009) ETOPO1 1 arc-minute global relief model: procedures, data sources and analysis. NOAA, technical memorandum, NESDIS, NGDC-24

Artemjev ME, Kaban MK, Kucherinenko VA, Demjanov GV, Taranov VA (1994) Subcrustal density inhomogeneities of the Northern Euroasia as derived from the gravity data and isostatic models of the lithosphere. Tectonophysics 240:248-280

Bagherbandi M, Sjöberg LE (2012) Non-isostatic effects on crustal thickness: a study using CRUST2.0 in Fennoscandia. Phys Earth Planet Inter 200-201:37-44 
Bassin C, Laske G, Masters G (2000) The current limits of resolution for surface wave tomography in North America. EOS Trans AGU 81:F897

Baudry N, Calmant S (1991) 3-D modeling of seamount topography from satellite altimetry. Geophys Res Lett 18:1143-1146

Bedle H, van der Lee S (2009) S velocity variations beneath North America. J Geophys Res 114:B07308

Bowin C, Scheer E, Smith W (1986) Depth estimates from ratio of gravity, geoid and gravity gradient anomalies. Geophysics 51(1):123-136

Braitenberg C, Zadro M (1999) Iterative 3D gravity inversion with integration of seismologic data. Boll Geofis Teor Appl 40(3/4):469-476

Braitenberg C, Wienecke S, Wang Y (2006) Basement structures from satellite-derived gravity field: South China Sea ridge. J Geophys Res 111:B05407

Carlson RL, Raskin GS (1984) Density of the ocean crust. Nature 311:555-558

Cazenave A, Schaeffer P, Berge M, Brossier C (1996) High-resolution mean sea surface computed with altimeter data of ERS-1 (Geodetic Mission) and TOPEX-POSEIDON. Geophys J Int 125:696-704

Chen W, Tenzer R (2014) Harmonic coefficients of the Earth's Spectral Crustal Model 180_ESCM180. Earth Sci Inf. doi:10.1007/s12145-014-0155-5

Christensen NI, Mooney WD (1995) Seismic velocity structure and composition of the continental crust: a global view. J Geophys Res 100(B7):9761-9788

Coffin MF (1992) Emplacement and subsidence of Indian Ocean plateaus and submarine ridges. In: Duncan RA et al (ed) Synthesis of results from scientific drilling in the Indian Ocean. Geophysical Monograph, vol 70. AGU, Washington, DC, pp 115-126

Crough ST (1983) The correction for sediment loading on the seafloor. J Geophys Res 88(B8):6449-6454

Cutnell JD, Kenneth WJ (1995) Physics, 3rd edn. Wiley, New York

Dixon TH, Naraghi M, McNutt MK, Smith SM (1983) Bathymetric prediction from Seasat altimeter data. J Geophys Res 88:1563-1571

Dziewonski AM, Anderson DL (1981) Preliminary reference Earth model. Phys Earth Planet Inter 25:297-356

Dziewonski AM, Hales AL, Lapwood ER (1975) Parametrically simple Earth models consistent with geophysical data. Phys Earth Planet Inter 10:12

Eckhardt DH (1983) The gains of small circular, square and rectangular filters for surface waves on a sphere. Bull Geod 57:394-409

Gladkikh V, Tenzer R (2012) A mathematical model of the global ocean saltwater density distribution. Pure App Geophys 169:249-257

Grand SP (2002) Mantle shear-wave tomography and the fate of subducted slabs. Phil Trans R Soc Lond 360:2475-2491

Grand SP, van der Hilst RD, Widiyantoro S (1997) Global seismic tomography: a snapshot of convection in the Earth. GSA Today 1:1-7

Gung Y, Romanowicz B (2004) Q tomography of the upper mantle using three-component long-period waveforms. Geophys J Int 157:813-830

Hammer S (1963) Deep gravity interpretation by stripping. Geophysics 28:369-378

Hayes DE (1988) Age-depth relationships and depth anomalies in the southeast Indian Ocean and South Atlantic Ocean. J Geophys Res 93(B4):2937-2954

Heiskanen WH, Moritz H (1967) Physical geodesy. WH Freeman and Co, San Francisco

Hinze WJ (2003) Bouguer reduction density, why 2.67? Geophysics 68(5):1559-1560

Houser C, Masters G, Shearer P, Laske G (2008) Shear and compressional velocity models of the mantle from cluster analysis of long-period waveforms. Geophys J Int 174:195-212

James DE, Fouch MJ, Carlson RW, Roth JB (2011) Slab fragmentation, edge flow and the origin of the Yellowstone hotspot track. Earth Planet Sci Lett 311(1):124-135

Jung WY, Vogt PR (1992) Predicting bathymetry from Geosat-ERM and shipborne profiles in the South Atlantic ocean. Tectonophysics 210:235-253

Kaban MK, Schwintzer P, Artemieva IM, Mooney WD (2003) Density of the continental roots: compositional and thermal contributions. Earth Planet Sci Lett 209:53-69

Kaban MK, Schwintzer P, Reigber Ch (2004) A new isostatic model of the lithosphere and gravity field. J Geod 78:368-385

Kane KA, Hayes DE (1992) Tectonic corridors in the South Atlantic: evidence for long-lived mid-ocean ridge segmentation. J Geophys Res 97(12):17,317-17330

Kaula WM (1969) Earth's gravity field, relation to global tectonics. Science 169:982-985

Kaula WM (1972) Global gravity and tectonics. In: Robertson EC (ed) The nature of the solid Earth. McGraw-Hill, New York, pp 385-405 
Kennett BLN, Engdahl ER (1991) Travel times for global earthquake location and phase association. Geophys J Int 105:429-465

Kennett BLN, Engdahl ER, Buland R (1995) Constraints on seismic velocities in the Earth from travel times. Geophys J Int 122:108-124

Kustowski B, Ekstrm G, Dziewoski AM (2008a) The shear-wave velocity structure in the upper mantle beneath Eurasia. Geophys J Int 174:978-992

Kustowski B, Ekstrom G, Dziewonski AM (2008b) Anisotropic shear-wave velocity structure of the Earth's mantle: a global model. J Geophys Res 113:B06306

Lambeck K (1971) Comparison of surface gravity data with satellite data. Bull Geod 100:203-219

Laske G, Masters G, Ma Z, Pasyanos ME (2012) CRUST1.0: An updated global model of Earth's crust. Geophys Res Abs 14, EGU2012-3743-1, EGU General Assembly 2012

Le Pichon X, Talwani M (1969) Regional gravity anomalies n the Indian Ocean. Deep Sea Res 16:263-274

Lekic V, Romanowicz B (2011) Inferring upper-mantle structure by full waveform tomography with the spectral element method. Geophys J Int 185(2):799-831

Makhloof AA (2007) The use of topographic-isostatic mass information in geodetic application. Dissertation D98, Institute of Geodesy and Geoinformation, Bonn

Mayer-Guerr T, Rieser D, Höck E, Brockmann JM, Schuh W-D, Krasbutter I, Kusche J, Maier A, Krauss S, Hausleitner W, Baur O, Jäggi A, Meyer U, Prange L, Pail R, Fecher T, Gruber T (2012) The new combined satellite only model GOCO03 s. Abstract, GGHS2012, Venice

Megnin Ch, Romanowicz B (2000) The shear velocity structure of the mantle from the inversion of of body, surface and higher modes waveforms. Geophys J Int 143:709-728

Montagner JP, Kennett BLN (1995) How to reconcile body-wave and normal-mode reference Earth models? Geophys J Int 125:229-248

Mooney WD, Laske G, Masters TG (1998) CRUST 5.1: a global crustal model at $5^{\circ} \times 5^{\circ}$. J Geophys Res 103B:727-747

Moritz H (2000) Geodetic reference system 1980. J Geod 74:128-162

Nataf HC, Ricard Y (1996) 3SMAC: an a priori tomographic model of the upper mantle based on geophysical modeling. Phys Earth Planet Int 95:101-122

Novák P (2010) High resolution constituents of the Earth gravitational field. Surv Geoph 31(1):1-21

Obrebski M, Allen RM, Xue M, Hung S-H (2010) Slab-plume interaction beneath the Pacific Northwest. Geophys Res Lett 37:L14305

Obrebski M, Allen RM, Pollitz F, Hung S-H (2011) Lithosphere-asthenosphere interaction beneath the western United States from the joint inversion of body-wave travel times and surface-wave phase velocities. Geophys J Int 185:1003-1021

Panning MP, Romanowicz BA (2006) A three dimensional radially anisotropic model of shear velocity in the whole mantle. Geophys J Int 167:361-379

Panning MP, Lekic V, Romanowicz BA (2010) Importance of crustal corrections in the development of a new global model of radial anisotropy. J Geophys Res 115:B12325

Parsons B, Sclater JG (1977) An analysis of the variation of the ocean floor bathymetry and heat flow with age. J Geophys Res 82:803-827

Pavlis NK, Factor JK, Holmes SA (2007) Terrain-related gravimetric quantities computed for the next EGM. In: Kiliçoglu A, Forsberg R (eds) Gravity field of the earth. Proceedings of the 1st international symposium of the international gravity field service (IGFS), Harita Dergisi, special issue, 18, General Command of Mapping, Ankara, Turkey

Pearson K (1895) Notes on regression and inheritance in the case of two parents. Proc R Soc Lond 58:240-242

Peltier WR (2007) Mantle dynamics and the D-double prime layer implications of the post-perovskite phase. In: Hirose K, Brodholt J, Lay T, Yuen D (eds) Post-perovskite: the last mantle phase transition, vol 174. AGU Geophysical Monograph, American Geophysics Union, pp 217-227

Phillips R, Lambeck K (1980) Gravity fields of the terrestrial planets: long-wavelength anomalies and tectonics. Rev Geophys Space Phys 18:27-76

Porritt RW, Allen RM, Boyarko DC, Brudzinski MR (2011) Investigation of Cascadia segmentation with ambient noise tomography. Earth Planet Sci Lett 309(1-2):67-76

Pratt JH (1855) On the attraction of the Himalaya Mountains and of the elevated regions beyond upon the plumb-line in India. Trans Roy Soc (Lond) B 145:101-104

Renkin MA, Sclater JG (1988) Depth and age in the North Pacific. J Geophys Res 93(B4):2919-2935

Rogers N, Blake S, Burton K (2008) An introduction to our dynamic planet. Cambridge University Press, Cambridge

Sandwell DT, Smith WHF (1997) Marine gravity anomaly from Geosat and ERS-1 satellite altimetry. J Geophys Res 102:10,039-10054 
Sclater JG, Anderson RN, Bell ML (1971) The elevation of the ridges and the evolution of the central eastern Pacific. J Geophys Res 76:7883-7915

Sclater JG, Hellinger S, Tapscott C (1977) The paleobathymetry of the Atlantic Ocean from the Jurassic to the present. J Geol 85:509-552

Sclater JG, Meinke L, Bennett A, Murphy C (1985) The depth of the ocean through the Neogene. In: Kennett JP (ed) The Miocene Ocean, Geol Sot Am, pp 1-19

Simmons NA, Forte AM, Boschi L, Grand SP (2010) GyPSuM: a joint tomographic model of mantle density and seismic wave speeds. J Geophys Res 115:B12310

Simmons NA, Myers SC, Johannesson G, Matzel E (2012) LLNL-G3Dv3: global P wave tomography model for improved regional and teleseismic travel time prediction. J Geophys Res 117:B10302

Sjöberg LE (2009) Solving Vening Meinesz-Moritz inverse problem in isostasy. Geophys J Int 179(3):1527-1536

Sjöberg LE, Bagherbandi M (2011) A method of estimating the Moho density contrast with a tentative application by EGM08 and CRUST2.0. Acta Geophys 58:1-24

Smith WHF (1993) On the accuracy of digital bathymetry data. J Geophys Res 98:9591-9603

Sykes TJS (1996) A correction for sediment load upon the ocean floor: uniform versus varying sediment density estimations-implications for isostatic correction. Mar Geol 133:1-2:35-49

Talwani M (1970) Gravity. In: Maxwell AE (ed) The Sea 4(1). Wiley Interscience, New York, pp 251-297

Talwani M, Pichon X, Le (1969) Gravity field over the Atlantic Ocean. In: Hart PJ (ed) The Earth's crust and upper mantle, Monograph 12, American Geophysical Union, pp 341-351

Tenzer R, Gladkikh V (2014) Assessment of density variations of marine sediments with ocean and sediment depths. Sci World J ID 823296, p 9. doi:10.1155/2014/823296

Tenzer R, Hamayun, Vajda P (2009a) Global maps of the CRUST 2.0 crustal components stripped gravity disturbances. J Geophys Res 114:BO5408

Tenzer R, Vajda P, Hamayun (2009b) Global atmospheric corrections to the gravity field quantities. Contr Geophys Geod 39(3):221-236

Tenzer R, Hamayun, Vajda P (2009c) A global correlation of the step-wise consolidated crust-stripped gravity field quantities with the topography, bathymetry, and the CRUST 2.0 Moho boundary. Contrib Geophys Geod 39(2):133-147

Tenzer R, Novák P, Vajda P, Gladkikh V, Hamayun (2012a) Spectral harmonic analysis and synthesis of Earth's crust gravity field. Comput Geosci 16(1):193-207

Tenzer R, Gladkikh V, Vajda P, Novák P (2012b) Spatial and spectral analysis of refined gravity data for modelling the crust-mantle interface and mantle-lithosphere structure. Surv Geoph 33(5):817-839

Tenzer R, Novák P, Gladkikh V (2012c) The bathymetric stripping corrections to gravity field quantities for a depth-dependant model of the seawater density. Mar Geod 35:198-220

Tenzer R, Hamayun, Novák P, Gladkikh V, Vajda P (2012c) Global crust-mantle density contrast estimated from EGM2008, DTM2008, CRUST2.0, and ICE-5G. Pure Appl Geophys 169(9):1663-1678

Trabant C, Hutko AR, Bahavar M, Karstens R, Ahern T, Aster R (2012) Data products at the IRIS DMC: stepping-stones for research and other application, Seism Res Lett 83(6):846:854

Tsoulis D (2004a) Spherical harmonic analysis of the CRUST2.0 global crustal model. J Geod 78(1/2):7-11

Tsoulis D (2004b) Two Earth gravity models from the analysis of global crustal data. Zeitschrift für Vermessungswesen 129(5):311-316

Turcotte D, Schubert G (2002) Geodynamics, 2nd edn. Cambridge University Press, New York

van der Lee S, Frederiksen A (2005) Surface wave tomography applied to the North American upper mantle. In: Levander A, Nolet G (eds) AGU monograph, seismic earth: array analysis of broadband seismograms, pp 67-80

van der Lee S, Nolet G (1997) Upper mantle S-velocity structure of North America. J Geophys Res 102:22815-22838

van Gelderen M, Koop R (1997) The use of degree variances in satellite gradiometry. J Geod 71:337-343

Wang T, Lin J, Tucholke B, Chen YJ (2011) Crustal thickness anomalies in the North Atlantic Ocean basin from gravity analysis. Geochem Geophys Geosyst 2:Q0AE02

Watts AB (2001) Isostasy and flexure of the lithosphere. Cambridge University Press, Cambridge

Wessel P, Watts AB (1988) On the accuracy of marine gravity measurements. J Geophys Res 93:393-413

Wienecke S, Braitenberg C, Götze H-J (2007) A new analytical solution estimating the flexural rigidity in the Central Andes. Geophys J Int 169(3):789-794

Wild F, Heck B (2004) Effects of topographic and isostatic masses in satellite gravity gradiometry. In: Proceedings, second international GOCE user workshop GOCE. The geoid and oceanography, ESAESRIN, Frascati, Italy, March 8-10, 2004 (ESA SP-569, June 2004), CD-ROM

Williams DL (1975) On the thermal evolution of the oceanic lithosphere. Geophys Res Lett 2:321-323 